\title{
All-Optical Amplitude Multiplexing Through Fiber Parametric Interaction Between Binary Signals
}

\author{
Marcelo L. F. Abbade ${ }^{1}$, Jorge D. Marconi², \\ Eric A. M. Fagotto ${ }^{1}$, Felipe R. Barbosa ${ }^{3}$, \\ André L. A. Costa ${ }^{3}$, Iguatemi E. Fonseca ${ }^{4}$ and Edson Moschim ${ }^{3}$ \\ 1 Pontifícia Universidade Católica de Campinas \\ 2 Universidade Federal do $A B C$ \\ ${ }^{3}$ Universidade Estadual de Campinas \\ ${ }^{4}$ Universidade Federal da Paraíba \\ Brazil
}

\section{Introduction}

The high bandwidth and low attenuation provided by optical fibers has turned them into the most extensively deployed transmission medium in communication systems worldwide. This is especially the case for systems that utilize bit rates ranging from hundreds of Mbits/s to several $\mathrm{Tb} / \mathrm{s}$ and whose span extends from a few tens of kilometers to intercontinental scales. In fact, global networking with the present speed and quality could hardly exist without fibers, which transport more information than all the other transmission media commercially used today combined (Ramaswamy, 2010).

The interest in optical fibers goes far beyond their valuable characteristics for signal propagation. In particular, fiber nonlinearities have been widely considered for the implementation of several all-optical devices. For example, wavelength converters based on cross-phase modulation (XPM) (Olsson et al., 2000) and fiber four-wave mixing (FWM) (Inoue \& Toba, 1992) have been investigated. Dispersion compensators and all-optical regenerators FWM have also been implemented (Chavez Boggio et al., 2004a), as well as wide-band tunable amplifiers, known as fiber optic parametric amplifiers (FOPA), relying on third-order parametric processes with one (Hansryd \& Andrekson, 2001) and two highpower pumps have been demonstrated (Chavez Boggio et al., 2005b). Recent work (Jamshidifar et al., 2010) shows that fiber tunable filters and demultiplexers can be achieved through parametric interaction in specially designed optical fibers.

Fiber-based nonlinear devices may also be used for all-optical signal processing. In this area, there is a special interest on techniques that provide conversion between different modulation formats. In fact, recent works deal with this subject and propose ways of performing analog to digital (Brzozowski \& Sargent, 2001), digital to analog (Oda \& Maruta, 2006), non-return-to-zero (NRZ) to return-to-zero (RZ) (Mishina et al., 2007), multilevel to 
binary (Fagotto \& Abbade, 2010) and binary to multilevel conversions (Zhou et al., 2006; Lu \& Miyazaki, 1997), among others.

A strong motivation for pursuing such research is that different kinds of optical networks (long-haul, optical packet switching, access and so on) may coexist and need to exchange information with one another (Mishina et al., 2007). Since each of them may present different physical and logical characteristics, signals within the boundaries of each optical network are subject to different types of impairments. Therefore, a modulation format used to minimize the bit error rate (BER) of signals within a given network domain may not be appropriate for other domains. Consequently, all-optical devices that provide modulation format conversion capabilities may be highly attractive to play a major role at the interface between different optical networks.

In this chapter, we focus on four techniques that use fiber nonlinear effects to perform optical amplitude multiplexing (OAM) of binary signals into multi-amplitude ones. We begin by reviewing the fundamentals of FWM and parametric amplification (PA) in Section 2. Then, in Section 3, we discuss two techniques that use FWM (Abbade et al., 2005; Abbade et al., 2006a; Abbade et al., 2006b) and PA (Abbade et al., 2010a; Abbade et al., 2010b; Marconi et al., 2011) to convert two 2-ASK signals into a quaternary amplitude-shift keying (4-ASK) one; named, respectively, OAM-4F and OAM-4P. In Section 4, utilization of FWM and PA (Abbade et al., 2011) to convert two binary signals into a ternary amplitude-shift keying (3-ASK) is approached. Such techniques are, respectively, termed OAM-3F and OAM-3P. It should be noted that OAM-3F is an innovation, presented for the first time in this work. Advantages of multi-amplitude modulation formats encompass higher tolerance to degradations caused by chromatic dispersion and the possibility of transmitting simultaneously two signals within the same optical bandwidth. Applications and a detailed comparison among the four techniques are presented in Section 5. Finally, conclusions are drawn in Section 6.

\section{Theory of parametric interactions in optical fibers}

An external electric field $\bar{E}$ applied to an optical fiber will cause an induced polarization $\bar{P}$ in the medium that will depend on its electrical susceptibility $\chi$. However, such a dependence will not only rely on the first-order susceptibility $\chi^{(1)}$, but also on higher-order terms. Processes relying on $\chi^{(2)}$ and $\chi^{(3)}$ are known, respectively, as second- and thirdorder parametric processes.

For an isotropic medium, the second order susceptibility $\underline{\chi}_{2}^{(2)}$ is equal to zero (in dipole approximation), which means that the term proportional to $\bar{E}^{2}$ vanishes. Therefore, only the contribution from the third order susceptibility $\chi^{(3)}$, proportional to $\bar{E}^{3}$ will remain. Thus, the polarization can be expressed as,

$$
\bar{P}=\varepsilon_{0}\left(\chi^{(1)} \bar{E}+\chi^{(3)} \bar{E}^{3}\right),
$$

where $\varepsilon_{0}$ is the vacuum electric permittivity.

At this point it is important to comment on two aspects of Eq. (1). First, the notation $\bar{E}^{3}$ is used as a simple way of writing the triple external product $\bar{E} \otimes \bar{E} \otimes \bar{E}$, which results in a third rank tensor with 27 elements. Second, the third order susceptibility $\chi^{(3)}$ is a fourth rank tensor which contains 81 independent elements, but it should be noted that the inner 
product of the $\chi^{(3)}$ tensor and the $\bar{E} \otimes \bar{E} \otimes \bar{E}$ tensor leads a simple vector. Furthermore, considering that silica glass is an isotropic material, the number of independent elements of the $\chi^{(3)}$ tensor is reduced to three independent elements by symmetry (Buck, 2005; Butcher \& Cotter, 1990). In addition, if the operating frequency range is far from resonances, then the number of independent elements is finally reduced to one (Buck, 2005). The "cubic" term of Eq. (1) is responsible for several nonlinear effects in optical fibers such as self-phase modulation (SPM), XPM, and FWM. It will be seen that, under proper conditions, FWM can be used to amplify a weak signal that propagates through an optical fiber along with a strong signal. To show that, let us start with the wave equation (Buck, 2005) for silica, a nonmagnetic material without free charges and currents,

$$
\nabla^{2} \bar{E}=\mu_{0} \frac{\partial \bar{\nabla} \times \bar{H}}{\partial t}=\mu_{0}\left(\varepsilon_{0} \frac{\partial^{2} \bar{E}}{\partial t^{2}}+\frac{\partial^{2} \bar{P}}{\partial t^{2}}\right) .
$$

where $\mu_{0}$ is the vacuum magnetic permeability, and $\bar{H}$ is the magnetic field (Jackson, 1998) that is related with the magnetic induction field $\bar{B}$ as $\bar{B}=\mu_{0} \bar{H}$ (then $\bar{\nabla} \times \bar{B}=\mu_{0} \bar{\nabla} \times \bar{H}$ ). Assuming that $\bar{J}=\partial \bar{P} / \partial t$, then the total polarization can be written as a sum of two terms, the linear and the nonlinear polarizations: $\bar{P}=\bar{P}_{L}+\bar{P}_{N L}$. Therefore, Eq. (2) can be written as

$$
\left(\nabla^{2}-\frac{n^{2}}{c^{2}} \frac{\partial^{2}}{\partial t^{2}}\right) \bar{E}=\mu_{0} \frac{\partial^{2} \overline{P_{N L}}}{\partial t^{2}},
$$

where $(n / c)^{2}=\left(\mu_{0} \varepsilon_{0}\left(1+\chi^{(1)}\right)\right)$, being $n$ the refractive index and $c$ the speed of light in vacuum. In the case of single mode fibers that are used in parametric devices, we have that $\Delta=\left(n_{\text {core }}-n_{\text {cladding }}\right) / n_{\text {cladding }}<<1$, where $n_{\text {core }}$ and $n_{\text {cladding }}$ are the refractive indexes of the core and the cladding, respectively (Agrawal, 2001).

Considering a typical value $\Delta \sim 0.003$, for weakly guiding fibers (Gloge, 1971), the longitudinal components of the electric fields are of the order of $\Delta^{1 / 2}$, which means that they are $\sim 20$ times smaller than the transversal components; therefore, they can be neglected in most practical applications. Then, considering that the fiber propagation is along the $z$-axis, the fields can be written as:

$$
\bar{E}_{L}=\hat{x} \psi(x, y) \frac{1}{2}\left[A_{L}(z) e^{-i\left(\beta\left(\omega_{L}\right) z-\omega_{L} t\right)}+c . c .\right],
$$

where c.c. stands for the complex conjugate of the previous term, $A_{L}(z)$ is the complex amplitude of the electric field, $\beta\left(\omega_{\mathrm{L}}\right)$ is the propagation constant for the angular frequency $\omega_{\mathrm{L}}$, and $\psi(x, y)$ is the transverse distribution of the electric field:

$$
\psi(x, y) \propto \begin{cases}A J_{0}\left(\kappa_{T} \rho\right) & \rho \leq a \\ B K_{0}\left(\gamma_{T} \rho\right) & \rho>a\end{cases}
$$

where $\mathrm{A}=\left[J_{0}\left(\kappa_{T} a\right)\right]^{-1}$ and $\mathrm{B}=\left[\mathrm{K}_{0}\left(\gamma_{\mathrm{T}} a\right)\right]^{-1}, \kappa_{T}=n_{\text {core }}^{2} k_{0}^{2}-\beta^{2}, \gamma_{T}=\beta^{2}-n_{\text {cladding }}^{2} k_{0}^{2}, k_{0}=2 \pi / \lambda$, $a$ is the core radius, and $\rho^{2}=x^{2}+y^{2}$. Here $J_{0}$ and $K_{0}$ are the Bessel functions corresponding to the fundamental mode (called $H E_{11}$ or $L P_{01}$ ) which is the only one propagating in singlemode fibers. 
As previously mentioned, the nonlinear process responsible for parametric amplification is the FWM. To show this let us assume that the refractive index can be written as the sum of a linear term and a nonlinear contribution, $n=n_{0}+I n_{2}$, where $n_{0}$ is the linear part of the refractive index and $n_{2}$ is the nonlinear refractive index (Boyd, 2008). The nonlinear contribution is proportional to the optical irradiance I (in the SI units system) (Boyd, 2008). Then, when two waves at angular frequencies $\omega_{1}$ and $\omega_{2}$ are launched together into an optical fiber, the refractive index will be modulated with a frequency $\left(\omega_{2}-\omega_{1}\right)$. Now, if a third wave at frequency $\omega_{3}$ is coupled along, a new wave at frequency $\omega_{4}=\omega_{3} \pm\left(\omega_{2}-\omega_{1}\right)$ will be generated. This new wave is called idler. The relation $\omega_{4}=\omega_{3} \pm\left(\omega_{2}-\omega_{1}\right)$ means that $\omega_{4}+$ $\omega_{1}=\omega_{3}+\omega_{2}$ and that $\omega_{4}+\omega_{2}=\omega_{3}+\omega_{1}$.

It is important to mention that when three waves are launched into the same fiber, a total of nine new frequencies can be in fact generated if all the combinations are taken into account (Hansryd et al., 2002). For instance, frequencies such as $\omega_{4}=2 \omega_{2}-\omega_{3}$ or $\omega_{4}=\omega_{3}-\omega_{2}+\omega_{1}$ are also possible. However, not all these frequencies are generated with the same efficiency. Generally calculations reckon only highly efficient processes and neglect the others. For instance, if we consider the case where $\omega_{1}<\omega_{3}=\omega_{2}$, we have that $2 \omega_{2}=\omega_{4}+\omega_{1}$; this process is a degenerate case of FWM that brings about parametric amplification when the wave at $\omega_{2}$ is a strong pump-signal and $\omega_{1}$ is a weak signal to be amplified. As a result, a new wave, an amplified copy of the signal at $\omega_{1}$, will be generated at $\omega_{4}$.

In order to standardize the notation, we shall denote the angular frequencies for the pump, the signal and the idler as $\omega_{\mathrm{p}}, \omega_{\mathrm{S}}$ and $\omega_{\mathrm{id}}$, respectively. Following this notation, the total electric field can be written as

$$
\begin{aligned}
\bar{E}=\bar{E}_{P}+\bar{E}_{S}+ & \bar{E}_{i d}=\hat{x} \psi(x, y) \frac{1}{2}\left[A_{P}(z) e^{-i\left(\beta\left(\omega_{P}\right) z-\omega_{P} t\right)}+\right. \\
& +A_{S}(z) e^{-i\left(\beta\left(\omega_{S}\right) z-\omega_{S} t\right)}+ \\
+ & \left.A_{i d}(z) e^{-i\left(\beta\left(\omega_{i d}\right) z-\omega_{i d} t\right)}\right]+c . c .
\end{aligned}
$$

where all the waves are supposed to have the same mode profile $\psi(x, y)$ and the same polarization on the $x$-axis. When this total electric field is included in Eq. (3), the Laplacian leads to the following electric fields (pump, signal, and idler)

$$
\begin{aligned}
\frac{\partial^{2} \overline{E_{j}}}{\partial z^{2}} & =\hat{x} \psi(x, y) \frac{1}{2}\left[\frac{\partial^{2} A_{j}(z)}{\partial z^{2}} e^{-i\left(\beta\left(\omega_{j}\right) z-\omega_{j} t\right)}-\right. \\
& -2 i \beta\left(\omega_{j}\right) \frac{A_{j}(z)}{\partial z} e^{-i\left(\beta\left(\omega_{j}\right) z-\omega_{j} t\right)}- \\
& \left.-\beta^{2}\left(\omega_{j}\right) A_{j}(z) e^{-i\left(\beta\left(\omega_{j}\right) z-\omega_{j} t\right)}\right]+ \text { c.c. }
\end{aligned}
$$

where $j=P$, $S$ or $i d$.

The slowly varying envelope approximation is introduced at this point, and is given by

$$
\left|\frac{\partial^{2} A_{j}}{\partial z^{2}}\right| \ll\left|\beta_{j} \frac{\partial A_{j}}{\partial z}\right|,
$$


or, considering that $\beta_{j}=\beta\left(\omega_{j}\right)=2 \pi / \lambda_{j}$, with $\lambda_{j}=2 \pi c / \omega_{j}$,

$$
\left|\lambda_{j} \frac{\partial}{\partial z}\left(\frac{\partial A_{j}}{\partial z}\right)\right|<<\left|\frac{\partial A_{j}}{\partial z}\right|,
$$

which means that the slopes of the envelope fields do not vary significantly along a wavelength distance $\left(\lambda_{j}\right)$ as compared to the envelope magnitude (Buck, 2005). Using this approximation, valid for most practical cases, the term with the second derivative of $z$ can be neglected.

Considering all these conditions and neglecting fiber attenuation, it is possible to obtain a set of three coupled equations for the three electric field amplitudes,

$$
\begin{gathered}
\frac{d A_{P}}{d z}=i \gamma\left[\left(\left|A_{P}\right|^{2}+2\left(\left|A_{S}\right|^{2}+\left|A_{i d}\right|^{2}\right)\right) A_{P}+2 A_{S} A_{i d} A_{P}^{*} \exp (i \Delta \beta z)\right], \\
\frac{d A_{S}}{d z}=i \gamma\left[\left(\left|A_{S}\right|^{2}+2\left(\left|A_{i d}\right|^{2}+\left|A_{P}\right|^{2}\right)\right) A_{S}+A_{i d}^{*} A_{P}^{2} \exp (-i \Delta \beta z)\right], \\
\frac{d A_{i d}}{d z}=i \gamma\left[\left(\left|A_{i d}\right|^{2}+2\left(\left|A_{S}\right|^{2}+\left|A_{P}\right|^{2}\right)\right) A_{i d}+A_{S}^{*} A_{P}{ }^{2} \exp (-i \Delta \beta z)\right],
\end{gathered}
$$

where the symbol $\left({ }^{*}\right)$ stands for the complex conjugate, $\Delta \beta$ is the linear phase mismatch $\Delta \beta=\beta\left(\omega_{s}\right)+\beta\left(\omega_{i d}\right)-2 \beta\left(\omega_{P}\right)$, and $\gamma=n_{2} \omega / c A_{e f f}$ is the fiber nonlinear coefficient. Here $n_{2}$ is the nonlinear refractive index which is related to $\chi^{(3)}$ as $n_{2}=3 \chi^{(3)} / 4 \varepsilon_{0} c n_{0}^{2}$, and $A_{\text {eff }}$ is the effective area (Agrawal, 2001). Note that the right-hand side of Eqs. (11)-(13) includes the terms of SPM, XPM and FWM.

The exact solutions of Eqs. (10)-(12) involve Jacobian elliptical functions as shown in (Chen, 1989). Here we follow an approximate solution that allows us to obtain a simple expression for the parametric gain. This approximation considers that the intensity of the pump is much higher than that of the signal and the idler. Therefore, the energy transferred from the pump to the signal (and the idler) can be considered negligible. For instance, if the ratio between the pump power and the signal power at the fiber input is $\sim 10^{4}-10^{5}$, and the signal gain is $\sim 20-25 \mathrm{~dB}$ at the fiber output, the signal (idler) power is still less than $1 \%$ of the pump power, which justifies the approximation.

Under such conditions Eqs.(10)-(12) may be written as,

$$
\begin{gathered}
\frac{d A_{P}}{d z}=i \gamma\left(P_{p} A_{P}\right), \\
\frac{d A_{S}}{d z}=i \gamma\left[2 P_{p} A_{S}+A_{i d}^{*} A_{P}^{2} \exp (-i \Delta \beta z)\right], \\
\frac{d A_{i d}}{d z}=i \gamma\left[2 P_{p} A_{i d}+A_{S}{ }^{*} A_{i d}{ }^{2} \exp (-i \Delta \beta z)\right],
\end{gathered}
$$


where $P_{P}=\left|A_{P}\right|^{2}$ is the pump power. These equations, which are valid for an ideal fiber with attenuation coefficient $\alpha=0$, have an analytical solution for the signal gain and for the idler conversion efficiencies as,

$$
\begin{gathered}
\frac{P_{S}}{P_{S}(0)}=1+\left(\frac{x_{0} \sinh (x)}{x}\right)^{2}, \\
\frac{P_{i d}}{P_{S}(0)}=\left(\frac{x_{0} \sinh (x)}{x}\right)^{2},
\end{gathered}
$$

where $P_{S}=\left|A_{S}\right|^{2}$ is the signal power at the fiber output, $P_{S}(0)$ is the signal power at the fiber input, $P_{i d}=\left|A_{i d}\right|^{2}$ is the idler power at the fiber output, $L$ is the fiber length, $x_{0}=\gamma P_{P} L$, $x=x_{0} \sqrt{1-\left(\Delta \beta_{T} / 2 \gamma P_{P}\right)^{2}}$, and $\Delta \beta_{T}=\Delta \beta+2 \gamma P_{P}$.

It should be noted that even when $\alpha \neq 0$, previous equations are good approximations if the pump power $P_{P}$ is replaced by $\overline{P_{P}}=\frac{1}{L} \int_{0}^{L} P_{P}(z) d z=P_{P}(0)\left(1-e^{-\alpha L}\right) / \alpha L$. The phase mismatch $\Delta \beta$ can be calculated by expanding $\beta(\omega)$ in Taylor series around an arbitrary frequency $\omega_{t}$ as follows:

$$
\begin{aligned}
& \beta(\omega)=\beta\left(\omega_{t}\right)+\left(\frac{\partial \beta}{\partial \omega}\right)_{\omega=\omega_{t}}\left(\omega-\omega_{t}\right)+\frac{1}{2}\left(\frac{\partial^{2} \beta}{\partial \omega^{2}}\right)_{\omega=\omega_{t}}\left(\omega-\omega_{t}\right)^{2}+ \\
& +\frac{1}{6}\left(\frac{\partial^{3} \beta}{\partial \omega^{3}}\right)_{\omega=\omega_{t}}\left(\omega-\omega_{t}\right)^{3}+\frac{1}{24}\left(\frac{\partial^{4} \beta}{\partial \omega^{4}}\right)_{\omega=\omega_{t}}\left(\omega-\omega_{t}\right)^{4}+\ldots \ldots \ldots . .
\end{aligned}
$$

Keeping terms up to the fourth order and taking $\omega_{t}=\omega_{\mathrm{P}}$ then,

$$
\Delta \beta(\omega)=\beta_{2}\left(\omega_{P}\right)\left(\omega-\omega_{P}\right)^{2}+\frac{\beta_{4}\left(\omega_{P}\right)}{12}\left(\omega-\omega_{P}\right)^{4} .
$$

Within the spectral region where the parameter $x$ is real, the parametric gain is maximum when $\Delta \beta_{T}=0\left(x=x_{0}\right)$, and its value is $G_{\max }=1+\sinh ^{2}\left(x_{0}\right)$. On the other hand, the gain has a local minimum (within the region of interest) when $\Delta \beta=0$, and its value is $G_{\min }=1+x_{0}^{2}$ (Chavez Boggio et al., 2005a). In other words, the parametric gain will be high if $\Delta \beta_{T}$ is small. This means that the pump must be tuned at some frequency within the fiber anomalous dispersion region, that is, $\omega_{P}<\omega_{0}$, with $\omega_{0}=2 \pi c / \lambda_{0}, \lambda_{0}$ the fiber zero dispersion wavelength and $c$ is the vacuum light speed. If the approximation $\beta_{4} \sim 0$ is valid, then the gain bandwidth can be roughly written as $\Delta \Omega=\omega_{P} \pm \sqrt{2 \gamma P_{P} /\left|\beta_{2}\right|}$.

In the extreme case of $\gamma P_{p}<<\beta$ and $P_{P} \sim P_{S}>>P_{i d}$, Eq. (17) gives the mixing condition without amplification (Stolen \& Bjorkholm, 1982). Here we change our notation and designate, the pump, the signal, and the idler powers as $P_{1}, P_{2}$, and $P_{-,+}$, and the angular 
frequencies change from $\omega_{P}, \omega_{S}$, and $\omega_{i d}$ to $\omega_{1}, \omega_{2}$, and $\omega_{-,+}$, respectively. The extreme case just considered implies that $\Delta \beta_{\mathrm{T}} \cong \Delta \beta, x \cong i L \Delta \beta$, and then $\sinh (x) \cong 2 i \sin \Delta \beta L$. Introducing all these results in Eq.(17) we have that

$$
P_{-,+}=4 P_{1,2}^{2} P_{2,1} \gamma^{2} L^{2}\left(\frac{\sin (\Delta \beta L)}{\Delta \beta L}\right)^{2} .
$$

The notation $P_{-,+}$refers to the power of the two principal FWM processes that generate waves at frequencies $\omega_{-,+}=2 \omega_{1,2}-\omega_{2,1}$.

\section{All-optical generation of quaternary amplitude-shift keying signals}

This section presents two all-optical techniques for multiplexing two binary ASK signals (ASK-2), traveling at different carrier wavelengths, in a single 4-ASK signal. In the first case, $\mathrm{OAM}-4 \mathrm{~F}$, the four levels of the quaternary pattern are obtained when the two binary signals, which have similar optical power, interact through FWM. The theoretical calculations that allow estimation of the power of the quaternary levels are developed from Eq. (27). The second approach, OAM-4P, used to generate the single 4-ASK signal from two binary ASK signals is based on PA. In this case, one of the signals is a strong optical signal that acts as a pump, with the unusual characteristic of being modulated by binary information. OAM-4F is presented in Section 3.1 and OAM-4P is approached in Section 3.2.

\subsection{Optical amplitude multiplexing through fiber four-wave mixing}

\subsubsection{Theory}

The diagram shown in Fig. 1 illustrates the principle of OAM-4F. Two co-polarized input signals at $\omega_{1}$ and $\omega_{2}$ are coupled into a fiber, where they co-propagate through a medium that favors the occurrence of FWM. When the fiber attenuation coefficient $\alpha \neq 0$, Eq. (20) can be rewritten as:

$$
P_{-,+}=\eta \gamma^{2} P_{1,2}^{2} P_{2,1} \exp (-\alpha L)\left[\frac{1-\exp (-\alpha L)}{\alpha}\right]^{2},
$$

where $P_{1}, P_{2}, P_{-}$, and $P_{+}$are the respective optical powers of the channels at frequencies $\omega_{1}$, $\omega_{2}, \omega_{-}$, and $\omega_{+}, L$ is the fiber length, $\gamma$ is the fiber nonlinear coefficient, and $\eta$ is the wavelength and intensity-dependent FWM generation efficiency, well described in the literature (Mussot et al., 2007), which is given by:

$$
\eta=4 L^{2}\left(\frac{\sin (\Delta \beta L)}{\Delta \beta L}\right)^{2} .
$$

If the channels at $\omega_{1}$ and $\omega_{2}$ are codified with ideal on-off keying (OOK) modulation, then $P_{-,+}$is null whenever one of these input channels transmits a 0-bit. Here, however, we assume that these channels are codified by a binary amplitude-shift keying (2-ASK) scheme, where the 0-bit powers of are intentionally offset. In this case, the extinction ratios (ER) corresponding to the channels at $\omega_{1}$ and $\omega_{2}$ are: 


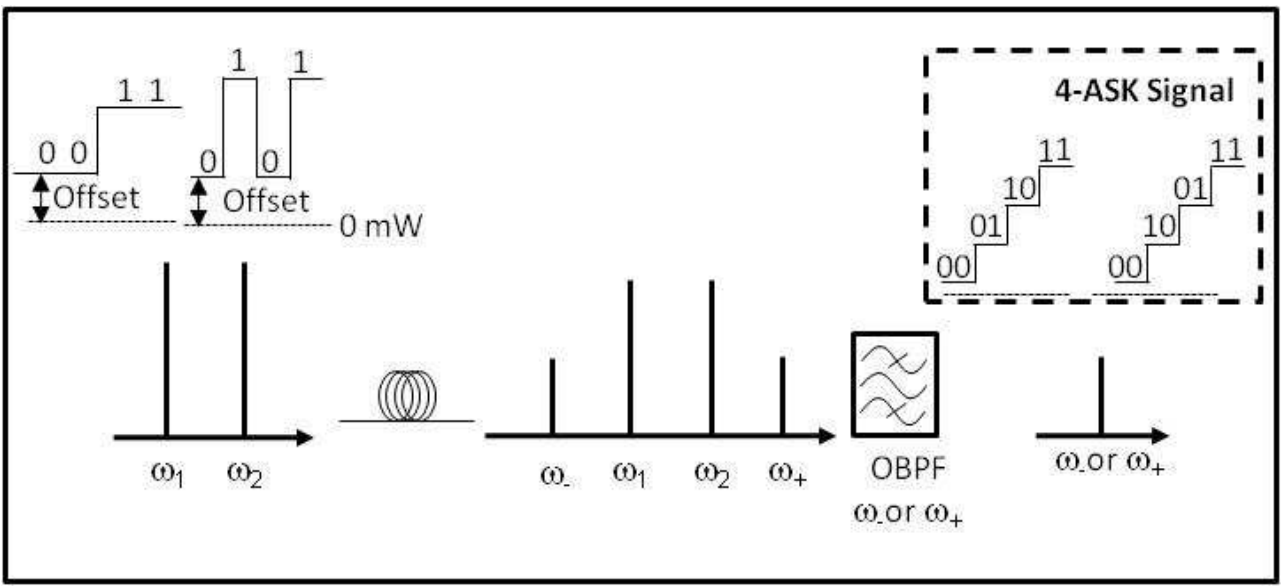

Fig. 1. Scheme illustrating the principle of operation of OAM-4F.

$$
\begin{aligned}
& r_{1}=P_{1}^{1}(0) / P_{1}^{0}(0) \\
& r_{2}=P_{2}^{1}(0) / P_{2}^{0}(0)
\end{aligned}
$$

where, $P_{i}^{j}(0)$ designates the power of bit $j(j=0$ or 1$)$ at the channel at $\omega_{i}(i=1$ or 2$)$. Eq. (21) indicates that the signals filtered at $\omega_{+}$may assume four different power levels given by:

$$
\begin{aligned}
& P_{\text {out }+}^{00}=k_{+} P_{1}^{0}(0)\left[P_{2}^{0}(0)\right]^{2} \\
& P_{\text {out }+}^{01}=k_{+} P_{1}^{0}(0)\left[P_{2}^{1}(0)\right]^{2} \\
& P_{\text {out }+}^{10}=k_{+} P_{1}^{1}(0)\left[P_{2}^{0}(0)\right]^{2} \\
& P_{\text {out }+}^{11}=k_{+} P_{1}^{1}(0)\left[P_{2}^{1}(0)\right]^{2}
\end{aligned}
$$

where $P_{\text {out }}^{m n}$ is the power of the signal envelope at $\omega_{+}$when the signal at $\omega_{1}$ transmits a bit $m$ $(m=0$ or 1$)$ and the signal at $\omega_{2}$ carries a bit $n(n=0$ or 1$)$, and

$$
k_{+}=\eta \gamma^{2} \exp (-\alpha L)\left[\frac{1-\exp (-\alpha L)}{\alpha}\right]^{2}
$$

Eqs. (24) clearly show that the signal formed at the fiber output, and selected by the optical band-pass filter (OBPF) centered at $\omega_{+}$, is a quaternary amplitude-shift keying (4-ASK) one. It should be noted that $P_{\text {out }+}^{00}$ is always the lowest power whereas $P_{\text {out }+}^{11}$ is always the highest 
one. On the other hand, $P_{\text {out }+}^{01}$ may be lower or higher than $P_{\text {out }+}^{10}$ depending, respectively, on whether $P_{1}^{0}(0)\left[P_{2}^{1}(0)\right]^{2}<P_{1}^{1}(0)\left[P_{2}^{0}(0)\right]^{2}$ or $P_{1}^{0}(0)\left[P_{2}^{1}(0)\right]^{2}>P_{1}^{1}(0)\left[P_{2}^{0}(0)\right]^{2}$. In case $P_{1}^{0}(0)\left[P_{2}^{1}(0)\right]^{2}=P_{1}^{1}(0)\left[P_{2}^{0}(0)\right]^{2}$, the quaternary signal degenerates into a ternary one.

The four-levels of quaternary signals give rise to an eye-diagram structure that comprises three eyes. We identify the eye made up of the two lowest power levels with the subscript "low"; analogously the subscripts "int" and "up" are utilized for the eyes that involve the two intermediate and the two higher power levels, respectively. For OAM-F4, it is possible to find the relative extinction ratios (RER) of such eyes, $r_{\text {low }}, r_{\text {int }}$, and $r_{\text {up }}$, by substituting (32) in (33). When $P_{1}^{0}(0)\left[P_{2}^{1}(0)\right]^{2}<P_{1}^{1}(0)\left[P_{2}^{0}(0)\right]^{2}$ :

$$
\begin{gathered}
r_{\text {low }}=P_{\text {out }+}^{01} / P_{\text {out }+}^{00}=r_{2}^{2} \\
r_{\text {int }}=P_{\text {out }+}^{10} / P_{\text {out }+}^{01}=r_{1} / r_{2}^{2} \\
r_{\text {up }}=P_{\text {out }+}^{11} / P_{\text {out }+}^{10}=r_{2}^{2}
\end{gathered}
$$

Similarly, if $P_{1}^{0}(0)\left[P_{2}^{1}(0)\right]^{2}>P_{1}^{1}(0)\left[P_{2}^{0}(0)\right]^{2}$ :

$$
\begin{gathered}
r_{\text {low }}=P_{\text {out }+}^{10} / P_{\text {out }+}^{00}=r_{1}^{2} \\
r_{\text {int }}=P_{\text {out }+}^{01} / P_{\text {out }+}^{10}=r_{2} / r_{1}^{2} \\
r_{\text {up }}=P_{\text {out }+}^{11} / P_{\text {out }+}^{01}=r_{1}^{2}
\end{gathered}
$$

Eqs. (24), (26) and (27) reveal some important properties of the generated 4-ASK signal. First, its powers do not depend on the phase of the input signals. Second, the power level distribution depends solely on the ERs of the two input signals. Finally, such power level distribution cannot be arbitrarily chosen. For instance, in the case where Eq. (26) hold, if one increases $r_{2}$, both $r_{\text {low }}$ and $r_{u p}$ are enhanced; however, $r_{i n t}$ is simultaneously decreased.

The analysis above can be repeated for the signal at $\omega$. In this case, Eqs. (24)- (27) would be modified, but the general properties of the generated 4-ASK signal would not change. Such analysis is left for the interested reader.

It is important to understand how information of the input binary signals may be recovered from the quaternary-amplitude one. To achieve this goal, it is assumed that the 4-ASK signal is photodetected by a circuit such as the one illustrated in Fig. 2.

Initially the signal is optically amplified and filtered at $\omega_{+}$; then, it is photo-detected by a PIN photo-diode with responsivity $R_{S}$, low-pass filtered and submitted to an electronic 


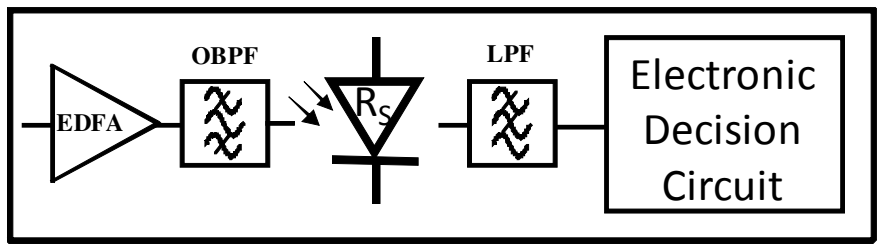

Fig. 2. Multiamplitude signal detector.

decision circuit (EDC), whose purpose is to indicate which bits were transmitted by the signals at $\omega_{1}$ and $\omega_{2}$. It is assumed that noise at EDC obeys a Gaussian distribution.

There are then two possibilities. The first one occurs when $P_{1}^{0}(0)\left[P_{2}^{1}(0)\right]^{2}<P_{1}^{1}(0)\left[P_{2}^{0}(0)\right]^{2}$ and it is illustrated in the left part of the inset of Fig. 1. A simple inspection of this figure indicates the following two detection rules should be utilized by the EDC: a) $\omega_{1}$ transmitted a bit 0 (1) whenever the two lower (upper) levels are detected; and b) $\omega_{2}$ transmitted a bit 0 (1) whenever the lower and third (second and fourth) power levels are detected.

These detection rules may be used to estimate the BERs of the binary signals extracted from the 4-ASK signals. To accomplish this goal, we first consider that the noise fluctuations between consecutive levels are much larger than the ones between non-consecutive levels. This hypothesis must hold for practical situations where even the noise fluctuations between adjacent levels must be low to keep the BERs at acceptable levels. Then, we observe from rule (a) and the left part of the inset of Fig. 1, that the BER for the signal at $\omega_{1}, B E R_{1}$, is equivalent to the one of a binary signal with threshold level between the second and third levels. In this way:

$$
B E R_{1}=Q\left(\frac{i_{10}-i_{01}}{\sigma_{10}+\sigma_{01}}\right)
$$

where, $Q(x)=(1 / \sqrt{2 \pi}) \int_{x}^{\infty} e^{-y^{2} / 2} d y$ is the complementary error function, $i_{x y}=R_{S} P_{\text {out }+}^{x y}$ is the average electronic current associated with power level $P_{\text {out }+}^{x y}$, and $\sigma_{x y}$ is the $i_{x y}$ standard deviation. From rule (b) and the left part of the inset of Fig. 2, it is observed that the BER for the signal at $\omega_{2}, B E R_{2}$, is equivalent to the average BER of three binary signals with decision thresholds between the first (lower) and second; the second and third; and the third and fourth (highest) levels:

$$
B E R_{2}=\frac{1}{3}\left[Q\left(\frac{i_{01}-i_{00}}{\sigma_{01}+\sigma_{00}}\right)+Q\left(\frac{i_{10}-i_{01}}{\sigma_{10}+\sigma_{01}}\right)+Q\left(\frac{i_{11}-i_{10}}{\sigma_{11}+\sigma_{10}}\right)\right]
$$

The second possibility occurs when $P_{1}^{0}(0)\left[P_{2}^{1}(0)\right]^{2}>P_{1}^{1}(0)\left[P_{2}^{0}(0)\right]^{2}$ and it is illustrated in the right part of the inset of Fig. 1. Following a procedure similar to the one described above and inspecting this figure, it is easy to verify that the detection rules are: c) $\omega_{1}$ transmitted a bit 0 (1) whenever the lower and third (second and fourth) power levels are detected and d) 
$\omega_{2}$ transmitted a bit 0 (1) whenever the two lower (upper) levels are detected. In this way, $B E R_{1}$ is now the average BER of the three eyes of the quaternary signal whereas $B_{2} R_{2}$ may be estimated from the BER of the intermediate eye:

$$
\begin{gathered}
B E R_{1}=\frac{1}{3}\left[Q\left(\frac{i_{10}-i_{00}}{\sigma_{10}+\sigma_{00}}\right)+Q\left(\frac{i_{01}-i_{10}}{\sigma_{01}+\sigma_{10}}\right)+Q\left(\frac{i_{11}-i_{01}}{\sigma_{11}+\sigma_{01}}\right)\right] \\
B E R_{2}=Q\left(\frac{i_{01}-i_{10}}{\sigma_{01}+\sigma_{10}}\right)
\end{gathered}
$$

We note that other reports suggest that the input binary signal may also be optically recovered from the 4-ASK signal with the use of transfer functions generated by self-phase modulation (Oda \& Maruta, 2006) or FWM (Fagotto \& Abbade, 2010) effects. However, a discussion concerning such all-optical approaches is beyond the scope of this chapter.

\subsubsection{Results and discussion}

Fig. 3 illustrates the experimental setup used to perform amplitude multiplexing through FWM. Two $1 \mathrm{~Gb} / \mathrm{s} 2^{12}-1$ pseudorandom bit sequences (PRBS) directly modulate the optical carriers at $f_{1}=193.00$ and $f_{2}=193.15 \mathrm{THz}$, where $f_{i}=2 \pi \omega_{i}(i=1,2)$. Previous to being coupled, these signals are co-polarized and then amplified by an Erbium-doped fiber amplifier (EDFA), EDFA1, up to an average peak power of $12 \mathrm{dBm}$. In the sequence, they are launched into a dispersion-shifted fiber (DSF) with $\alpha=0.20 \mathrm{~dB} / \mathrm{km}, \lambda_{0}=1550 \mathrm{~nm}$, dispersion slope $S_{0}=0.074 \mathrm{ps} /(\mathrm{nm} . \mathrm{km}), \gamma=2.0(\mathrm{~W} . \mathrm{km})^{-1}$, and $\mathrm{L}=25.0 \mathrm{~km}$. Since the powers at the fiber input are relatively low, it is not necessary to use any mechanism to prevent Brillouin backscattering.

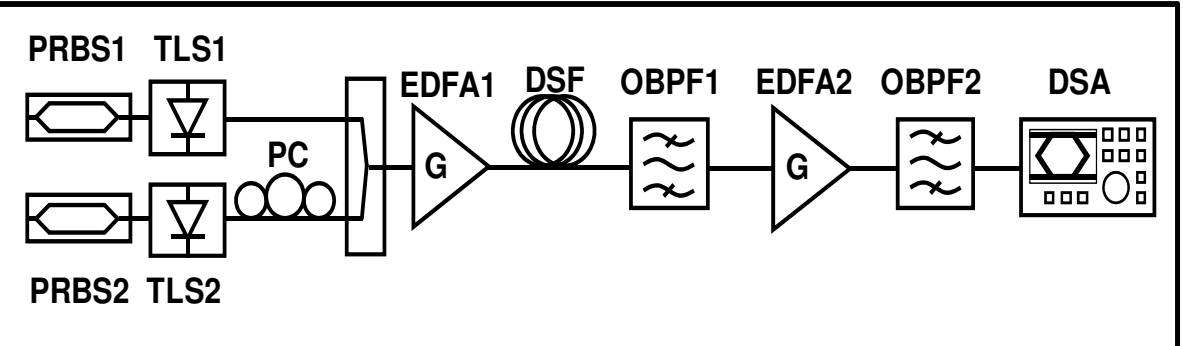

Fig. 3. Experimental setup for all-optical multiplexing.

Actually, since both signals are modulated, the Brillouin backscattering threshold should be a few $\mathrm{dB}$ higher than in the case of continuous-wave (cw) operation. Fiber FWM generates two sidebands, one at $f_{-}=192.85 \mathrm{THz}$ and other at $f_{+}=193.30 \mathrm{THz}$. The latter is filtered by OBPF1, amplified by EDFA2, and then filtered again by OBPF2. Next, the signal is received by a digital signal analyzer (DSA). The double filtering is required because the first filter OBPF1 is not enough to eliminate effectively the input signals. Therefore, the OBPF1 output needs to be amplified and then filtered again by OBPF2 before being inputted to the DSA. 
The signal power spectra at (a) EDFA1 input, (b) DSF output, and (c) OBPF2 output are plotted in Fig. 4. An optical signal-to-noise ratio (OSNR) of $27 \mathrm{~dB}$ is achieved at the output of the second optical filter.

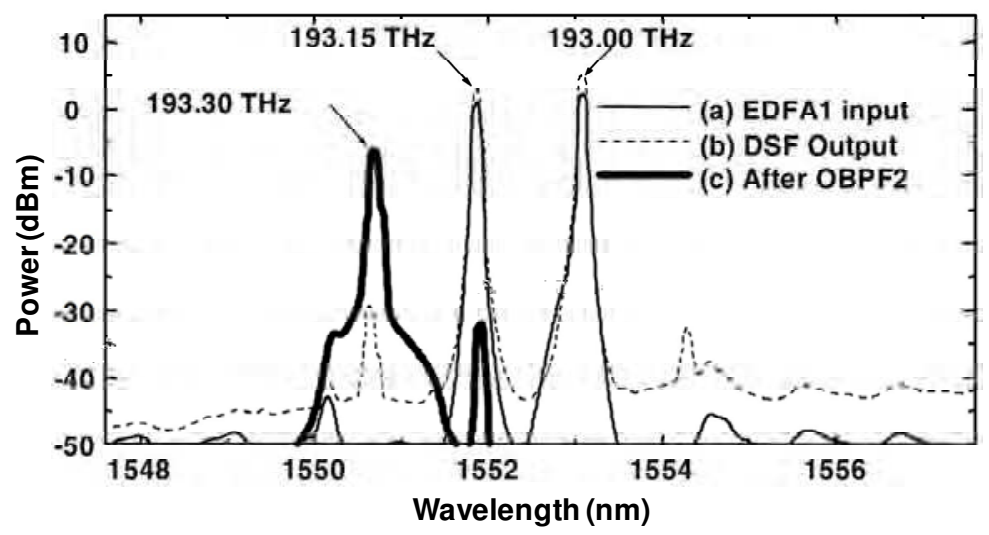

Fig. 4. Power Spectra.

Fig. 5 exhibits two unsynchronized PRBSs used to modulate the signals (a) at $f_{1}$ with $r_{1}=4.0$ $\mathrm{dB}$ and (b) at $f_{2}$ with $r_{2}=1.7 \mathrm{~dB}$, and the (c) quaternary signal obtained at $f_{+}$. In this situation, $r_{1}>r_{2}{ }^{2}$ (which is equivalent to $P_{1}^{0}(0)\left[P_{2}^{1}(0)\right]^{2}<P_{1}^{1}(0)\left[P_{2}^{0}(0)\right]^{2}$ ) and the quaternary signal is governed by (26).

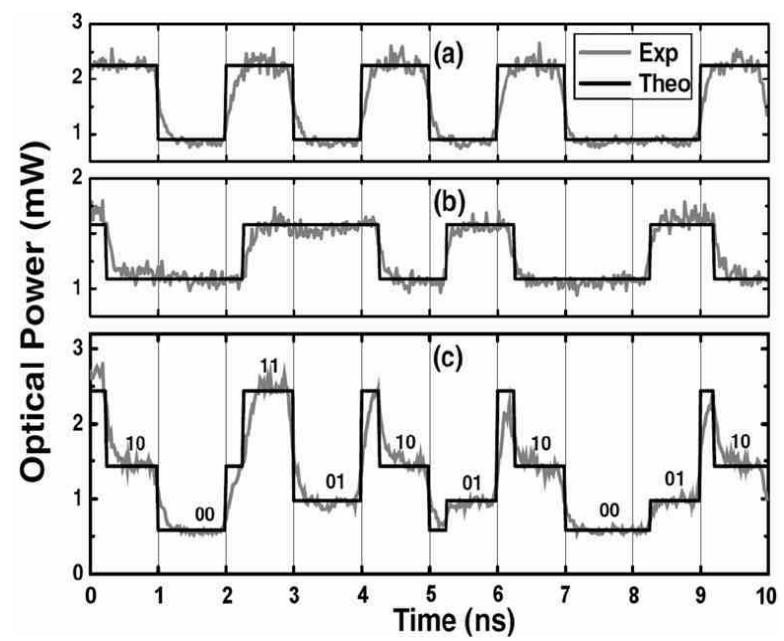

Fig. 5. (a) Binary input sequence at $f_{1}$ and (b) at $f_{2}$ and (c) quaternary output signal.

Fig. 6a shows the eye diagrams for $r_{2}=2.6 \mathrm{~dB}$, and $r_{1}=2.6 \mathrm{~dB}$. In this case, again $r_{1}{ }^{2}>r_{2}$ $\left(P_{1}^{0}(0)\left[P_{2}^{1}(0)\right]^{2}<P_{1}^{1}(0)\left[P_{2}^{0}(0)\right]^{2}\right)$ and so the two intermediate powers, in increasing 
magnitude of power, represent levels 01 and 10 (where, as before, level $i j$ stands for the bit $i$ transmitted by the channel at $\omega_{1}$ and for the bit $j$ transmitted by the channel at $\omega_{2}$ ). When $r_{1}$ is increased to $4.8 \mathrm{~dB}, r_{\text {int }}$ in $(26 \mathrm{~b})$ becomes close to unity and the two intermediate eyes get very close; this is shown in Fig. $6 \mathrm{~b}$. If $r_{1}$ is further increased to $7.7 \mathrm{~dB}$, then $r_{1}{ }^{2}<r_{2}$
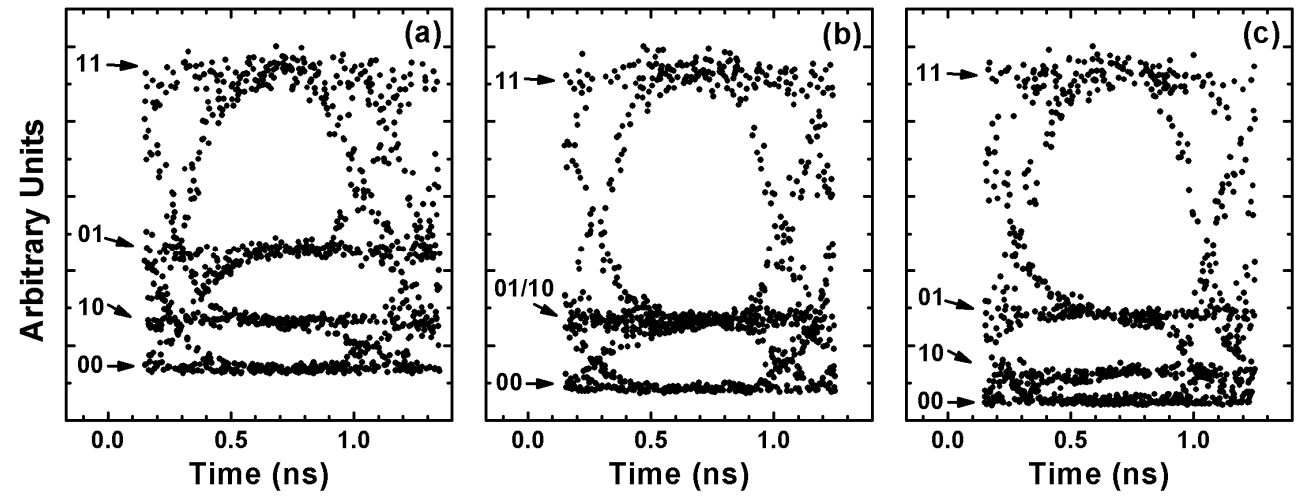

Fig. 6. Eye-diagrams for $r_{2}=2.6 \mathrm{~dB}$ and (a) $r_{1}=2.6 \mathrm{~dB}$, (b) $r_{1}=4.8 \mathrm{~dB}$, and (c) $r_{1}=7.7 \mathrm{~dB}$.

$\left(P_{1}^{0}(0)\left[P_{2}^{1}(0)\right]^{2}>P_{1}^{1}(0)\left[P_{2}^{0}(0)\right]^{2}\right)$ and the position between levels 01 and 10 is exchanged;

Fig 6(c) shows the eye diagrams for such situation.

To complete such analysis, graphs of (a) $r_{u p}$, (b) $r_{i n t}$, and (c) $r_{\text {low }}$ as a function of $r_{1}$ are plotted in Fig. 7 for experimental data and theoretical curves, for $r_{2}=2.6 \mathrm{~dB}$. The agreement between such results is quite good. As predicted by (27), increasing $r_{1}$, initially causes $r_{\text {int }}$ to decrease.
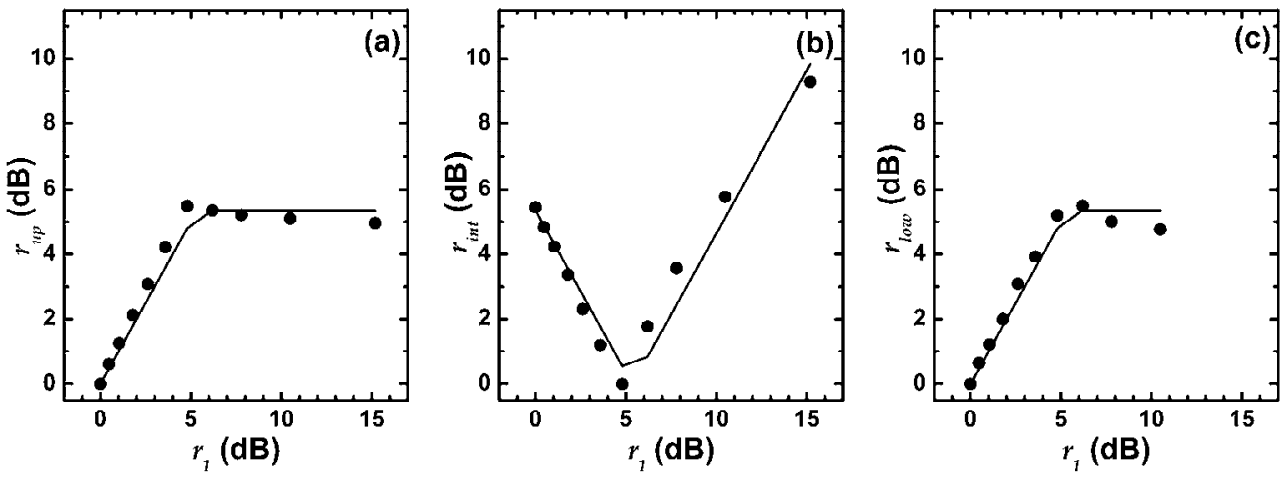

Fig. 7. Theoretical (line) and experimental (dots) ERs for the quaternary signal. When $r_{1} \approx r_{2}{ }^{2}$, $r_{\text {int }}$ achieves a minimum value; this is the point where the quaternary signal degenerates into a ternary-amplitude one and where levels 10 and 01 exchange their positions. As $r_{1}$ is further increased, (27) is no longer valid and (26) needs to be applied; in this region $r_{\text {int }}$ increases again. It is also observed that $r_{u p} \approx r_{\text {low }}$ for any value of $r_{1}$. As $r_{1}$ is increased, $r_{u p}$ and $r_{\text {low }}$ initially also increase, in agreement with (27). Then, for $r_{1} \geq r_{2}{ }^{2}, r_{u p}$ and $r_{\text {low }}$ remain at a constant and maximum value in agreement with (26). 
It should be noted that the FWM equations presented in Section 2 assume that the involved signals are in $c w$ regime. The accordance between theoretical results and experimental data observed in this subsection suggest that such FWM equations are also valid when the involved signals are modulated.

We reckon that the first experimental results concerning this technique were reported in $(\mathrm{Xu}$ et al., 2005). There, two $10 \mathrm{~Gb} / \mathrm{s}$ signals were multiplexed in a $20 \mathrm{~Gb} / \mathrm{s}$ 4-ASK one, which was then transmitted through a $160 \mathrm{~km}$ single mode fiber (SMF) link; after such propagation distance, chromatic dispersion was compensated. The eye-penalties experienced by the quaternary signal were relatively small. In another experiment (Abbade et al, 2006), a 2 $\mathrm{Gb} / \mathrm{s}$ 4-ASK was generated and propagated through a $20-\mathrm{km}$ long SMF link in a field-trial network (FAPESP, n.d.). Eqs. (28) and (29) were utilized to estimate the BERs for signals with different values of $r_{1}$ and $r_{2}$. Although chromatic dispersion was not compensated and no special care concerning the bandwidth of filters was taken into account, $B E R_{1}<10^{-9}$ and $B E R_{2}<10^{-12}$ were obtained for several combinations of values of $r_{1}$ and $r_{2}$. This indicates that the 4-ASK signal generated by FWM amplitude multiplexing could be properly utilized in practical applications.

BER performance of multi-amplitude signals depends on the dominant kind of noise. Generally speaking, different types of noise require different power level distributions to minimize signal BER. Theoretical and experimental analyses of such optimum distribution for 4-ASK signals are presented in (Walklin \& Conradi, 1999). In particular, it is shown that under the dominance of amplified spontaneous emission (ASE) noise, the power levels of a quaternary amplitude signal should be in proportions of 0: 1: 4: 9 .

Concerning the 4-ASK generated by OAM-4F, such optimal distribution would apply to the cases described by Eqs. (28b) and (29a), where consecutive power levels represent complementary bits (i.e., 0101). Unfortunately, the proportions of 0:1: 4: 9 cannot be achieved by FWM amplitude multiplexing. This may be understood by noting in Eq. (24a) that $P_{\text {out }+}^{00}$ is never null; moreover, $r_{u p}=r_{\text {low }}((26)$ and $(27))$. In the cases described by Eqs. (28a) and (29b), the two lowest power levels stand for bit 0 , whereas the two highest ones represent a bit 1 . Therefore, for a given average power, BER minimization would require the two lowest power levels and also the two highest power levels to coincide. But this degenerates the 4-ASK signal into a 2-ASK one, which does not allow information from the signals at $\omega_{1}$ and $\omega_{2}$ to be recovered. This discussion shows that minimizing $B E R_{1}$ does not lead to a minimization of $B E R_{2}$.

\subsection{Optical amplitude multiplexing through fiber parametric amplification}

\subsubsection{Theory}

As detailed in Section 2, fiber optics parametric amplifiers are realized by coupling both a weak probe signal at angular frequency $\omega_{S}$, and a high-power pump signal at angular frequency $\omega_{P}$ into an optical fiber that acts as a nonlinear medium. Usually the performance of parametric amplifiers is analyzed for binary signals with two intensity levels representing bits "0" and "1" (Kalogerakis et al., 2006; Mussot et al., 2007; Chavez Boggio et al., 2004b; Peucheret et al., 2006) as sketched in Fig. 8(a). The power of these levels at the fiber input are indicated by $P_{S}^{0}(0)$ and $P_{S}^{1}(0)$, respectively; the ideal $P_{S}^{0}(0)$ being null. Figure $8(\mathrm{~b})$ shows a 

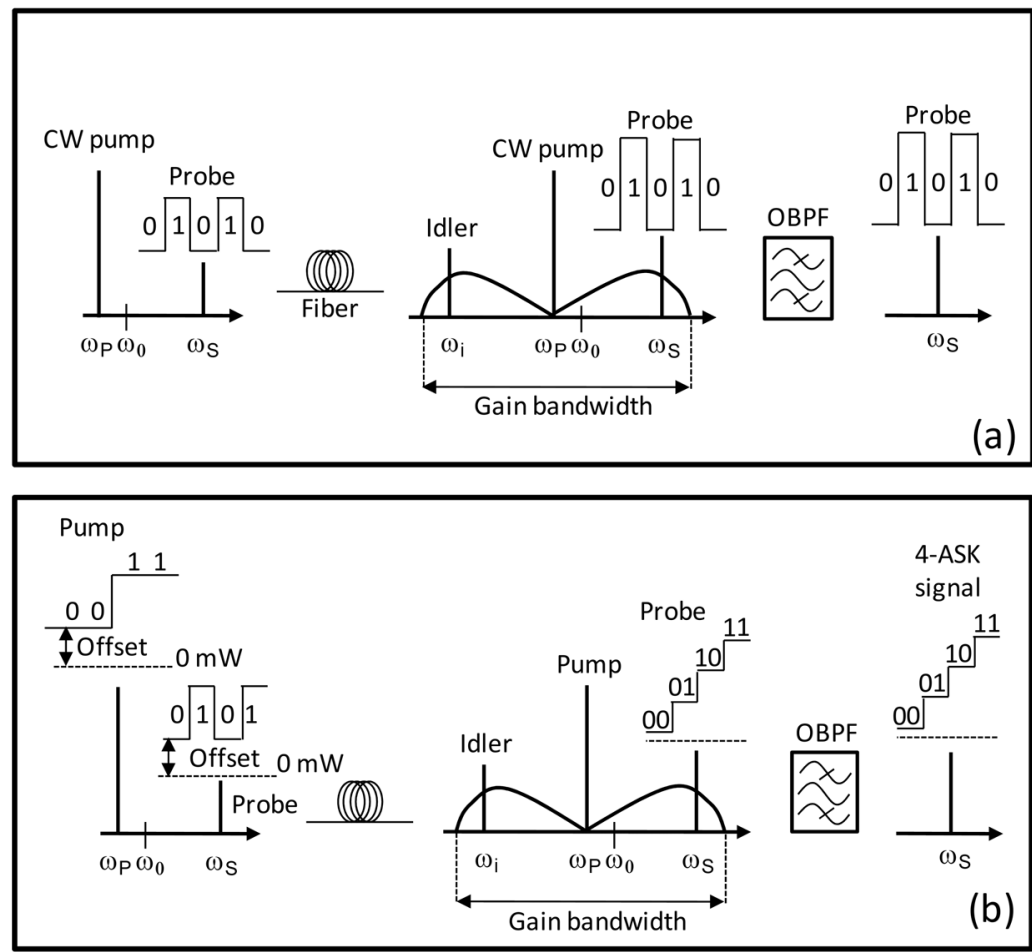

Fig. 8. Scheme for (a) PA and (b) ASK-4 generation. ( $\omega_{k}$ with $k=P, S, i$ represents the angular frequencies of the pump, the signal and the idler, and $\omega_{0}=2 \pi \mathrm{c} / \lambda_{0}$, where $\lambda_{0}$ is the zero dispersion wavelength of the fiber).

scheme of the theoretical principle of OAM-4P. Essentially, this principle is equal to the PA, but two new features are added to the signals involved.

First, as in OAM-4, the power of the probe signal is intentionally offset from zero. Thus, the ER of the signal is no longer infinite (in contrast to the ideal OOK signals), and is given by:

$$
r_{S}=P_{S}^{1}(0) / P_{S}^{0}(0) .
$$

Second, the pump is also modulated with binary information, in an analogous way to that of the probe signal. The powers of the " 0 " and " 1 " bits transmitted by the pump at the fiber input are designated as $P_{P}^{0}(0)$ and $P_{P}^{1}(0)$, respectively. It is assumed that $P_{P}^{0}(0)$ is considerably higher than $P_{S}^{1}(0)$. Once the pump is modulated, it will provide two amplification factors, $G_{P}^{0}$ or $G_{P}^{1}$, depending on whether the pump information is a " 0 " or " 1 " bit. Consequently, each of the binary levels of the signal at $\omega_{S}$ will have a lower or a higher gain, splitting into two new power levels at the fiber output.

For example, consider that the signal at $\omega_{S}$ has the " 1 " bit power level, then the pump modulation will split it in two power levels " 01 " and " 11 ", depending on whether the pump information is a bit " 0 " or " 1 ", respectively. This argument is also valid when the probe 
signal transports a bit " 0 " power level. In such case, the probe signal will either assume a " 00 " or " 10 " power level at the fiber output, depending again on whether the pump information is a bit " 0 " or " 1 ", respectively. Therefore, the power levels of the signal envelope at $\omega_{S}$ and at $z=L$ will be:

$$
\begin{aligned}
& P_{\text {out }}^{00}=G_{P}^{0} P_{S}^{0}(0) \\
& P_{\text {out }}^{01}=G_{P}^{0} P_{S}^{1}(0) \\
& P_{\text {out }}^{10}=G_{P}^{1} P_{S}^{0}(0) \\
& P_{\text {out }}^{11}=G_{P}^{1} P_{S}^{1}(0)
\end{aligned}
$$

where $P_{\text {out }}^{i j}$ represents the power of the signal at $\omega$ s and at $z=L$, when bits $i$ and $j(i, j=$ " 0 " or "1") at $z=0$ are assigned to the pump and the probe signal, respectively. $G_{P}^{0}$ and $G_{P}^{1}$ can be obtained through Eq. (16) with pump powers $P_{P}^{0}$ and $P_{P}^{1}$, respectively.

Note that Eqs. (31) clearly show that the 4-ASK signal contains information from both the pump and the probe signal. As in Section 3.1, we identify the eye made up of the two lowest power levels with the subscript "low"; analogously, the subscripts "int" and "up" are utilized for the eyes that involve the two intermediate and the two higher power levels, respectively. By using this convention and considering $P_{\text {out }}^{10} \geq P_{\text {out }}^{01}\left(G_{P}^{0} P_{S}^{1}(0) \leq G_{P}^{1} P_{S}^{0}(0)\right)$, the ERs for each one of these eyes can be written as:

$$
\begin{aligned}
& r_{\text {low }}=\frac{P_{\text {out }}^{01}}{P_{\text {out }}^{00}}=r_{S} \\
& r_{\text {int }}=\frac{P_{\text {out }}^{10}}{P_{\text {out }}^{01}}=\frac{r_{G}}{r_{S}} \\
& r_{\text {up }}=\frac{P_{\text {out }}^{11}}{P_{\text {out }}^{10}}=r_{S}
\end{aligned}
$$

where $r_{G}$ is defined as

$$
r_{G}=\frac{G_{P}^{1}}{G_{P}^{0}},
$$

and it is the ER associated with the amplification factors $G_{P}^{0}$ and $G_{P}^{1}$. In the case $P_{\text {out }}^{10} \leq P_{\text {out }}^{01}$ $\left(G_{P}^{0} P_{S}^{1}(0) \geq G_{P}^{1} P_{S}^{0}(0)\right)$, the ERs are given by:

$$
r_{\text {low }}=\frac{P_{o u t}^{10}}{P_{\text {out }}^{00}}=r_{G}
$$




$$
\begin{gathered}
r_{\text {int }}=\frac{P_{\text {out }}^{01}}{P_{\text {out }}^{10}}=\frac{r_{S}}{r_{G}} \\
r_{\text {up }}=\frac{P_{\text {out }}^{11}}{P_{\text {out }}^{01}}=r_{G}
\end{gathered}
$$

Eqs. (32) and (34) show that the power level distribution of the generated 4-ASK signal does not depend on the powers of the probe signal at $\omega_{\mathcal{S}}$ (only on its ER), but on the powers utilized in the pump. This is quite reasonable because in our analytical model the power of the probe signal was supposed to be much lower than the pump power, and so it was neglected (Song et al., 1999a). Besides that, it is clear that by controlling only the parameters $r_{S}$ and $r_{G}$ it is possible to regulate the power of the quaternary levels.

The pump ER $r_{P}=P_{P}^{1}(0) / P_{P}^{0}(0)$ is controlled by setting the values of the pump average powers, and then it is possible to control the value of $r_{G}$. For the ideal OOK modulation in the pump $\left(P_{P}^{0}(0)=0\right)$, Eqs. (31), (32) and (34) are still valid, and in this special case $G_{P}^{0}=1$ and $r_{G} \cong G_{P}^{1}$.

The theoretical model for 4-ASK presented here and applied to modulated signals is actually based on a set of equations generated for $c w$ signals. In the next two subsections the applicability of the technique to practical situations is tested through simulations and experiments, showing that our assumptions work fine.

\subsubsection{Results and discussions}

Fig. 9 shows the experimental setup used in both simulations and experiments. The lasers (two DFB lasers) that were used as the pump and the signal were tuned at $\lambda_{P}=1553.5 \mathrm{~nm}$ and $\lambda_{S}=1552.2 \mathrm{~nm}\left(\lambda_{k}=2 \pi c / \omega_{\mathrm{k}}, k=P\right.$ or $\left.S\right)$, respectively.

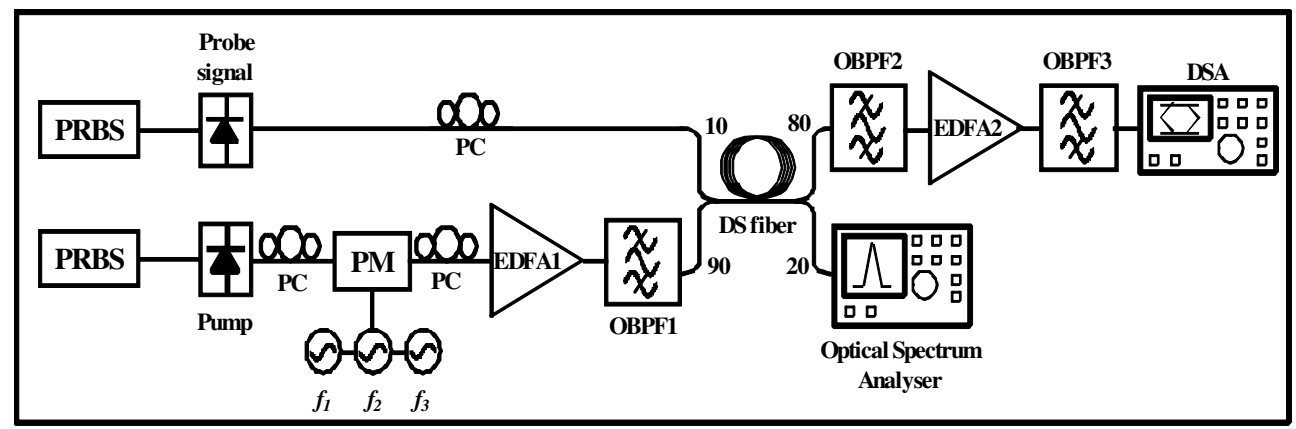

Fig. 9. Experimental setup.

A pseudo-random bit sequence at $1 \mathrm{~Gb} / \mathrm{s}$ was used to modulate the lasers through direct modulation. In order to suppress stimulated Brillouin scattering (SBS), the pump linewidth was broadened by phase modulation using a phase modulator (PM) driven by three RF signals. After the Erbium-doped fiber amplifier (EDFA 1), the pump was filtered with an 
optical band-pass filter (OBPF) to suppress most of the amplified spontaneous emission (ASE). A 90/10 coupler was used to couple the pump and the signal to a $7 \mathrm{~km}$ long segment of dispersion shifted fiber (DSF).

The other fiber parameters were: the zero-dispersion wavelength $\lambda_{0}=1550.2 \mathrm{~nm}$, the zerodispersion wavelength variation $\Delta \lambda_{0}=0.08 \mathrm{~nm}$, the dispersion slope $S_{0}=0.074 \mathrm{ps} / \mathrm{nm}^{2} / \mathrm{km}$, the nonlinear coefficient $\gamma=2.1 \mathrm{~W}^{-1} \mathrm{~km}^{-1}$, and the attenuation coefficient $\alpha=0.2 \mathrm{~dB} / \mathrm{km}$. The maximization of the parametric gain was achieved by aligning the pump and the signal states of polarization using polarization controllers (PCs). The optical power was divided with an optical coupler at the fiber output for spectral and systemic characterization. A fraction of $\sim 20 \%$ was delivered to an optical spectrum analyzer (OSA) for recording the spectra. The remaining power $(\sim 80 \%)$ was sent to an OBPF centered at the signal frequency $\omega_{s}$. The signal was then amplified and filtered again to reduce the ASE power accumulated in the amplification stage. Finally, the signal with an average power of approximately $0 \mathrm{dBm}$ entered a digital oscilloscope to characterize the resulting ASK-4 eye-diagrams. The quaternary signal was also analyzed after being transmitted through a $75 \mathrm{~km}$-long spool of standard fiber. To compensate for the dispersion due to the propagation, the signal was also passed through a $-68 \mathrm{ps} / \mathrm{nm}$ compensating fiber.

An accurate control of the ERs of both the pump and the signal was a necessary item for the proper evaluation of the proposed technique. Thus, with the equipment available in our labs, we found that the required accuracy was more easily obtained by programming the modulation index of directly modulated lasers than by varying the bias voltage of the external modulator. However, the technique should also hold for signals with external modulation if specific modulators were available. It is anticipated that due to the femto-second response of PA (Grudinin et al., 1987) the technique should also work well for higher bit rates.

The simulated results presented here were obtained using exactly the same setup as used for the experiments. To perform the simulations as close as possible to the experiments, the laser linewidth was set at the maximum value of the equipment at $\sim 30 \mathrm{MHz}$. Also the same phase modulation scheme used experimentally was added to our simulations. This point is particularly important because the PM induces noise at the level " 1 " of the binary eyediagrams. Consequently, additional noise is also expected at quaternary levels. The DSF was divided into 10 segments to perform the simulations in order to consider the influence of the zero dispersion wavelength variations along the fiber. All segments had the same length and the $\Delta \lambda_{0}$ was distributed within the range $\lambda_{0}-\Delta \lambda_{0} / 2$ to $\lambda_{0}+\Delta \lambda_{0} / 2$. The segments were randomly ordered. The propagation along each segment was handled by solving the nonlinear Schrödinger equation using the split-step Fourier method (Agrawal, 2001). A commercially available software program was used to perform the simulations.

Fig. 10 shows spectral and temporal results. Fig. 10(a) shows a comparison between the simulated and the experimental spectra at the fiber output. Note that the main difference between them is in the noise region of the spectra. The principal reason for such a difference is that the ASE originated in the pump Erbium booster amplifier, that is not completely suppressed by the band-pass filter, is then amplified by the parametric amplifier. It is important to note that $\omega_{S}$ is placed within the frequency region where the signal performance is not affected by the phase modulation used to suppress the SBS (Boggio et al., 2005a). This region for conventional (non-modulated pump) PA is the region of maximum 

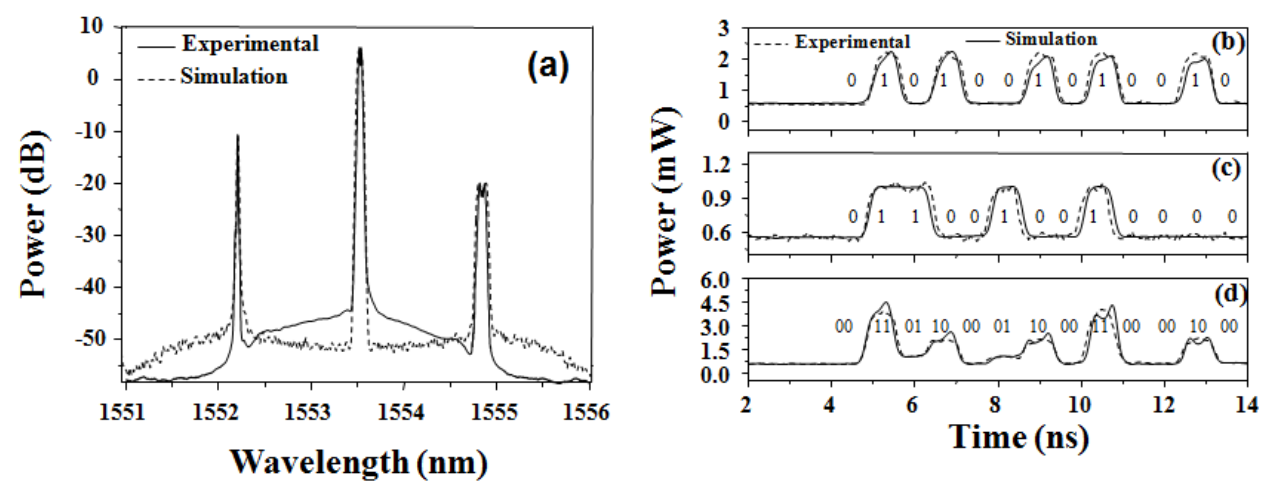

Fig. 10. (a) Experimental and simulated optical spectrum at the fiber output. (b) Binary pump. (c) Binary signal before amplification. (d) ASK-4 signal obtained after PA

gain; however, the gain bandwidth does not remain the same when the pump transmits either a bit " 1 " or " 0 ", thus the change in bandwidth also modifies the spectral regions where the PM affects the signal performance. Consequently, some influence on the OSNR performance of the quaternary signal is expected.

Two bit sequences carried by the pump and the probe signal at the fiber input are illustrated in Figs. 10(b) and (c). The resulting quaternary signal is illustrated in Fig. 10(d). The pump and the probe signal ERs are $r_{P}=6.0 \mathrm{~dB}$ and $r_{S}=2.6 \mathrm{~dB}$, respectively. As expected the signal of Fig. 10(d) presents four well-defined power levels. Note that the same power levels are obtained when the bits of the pump and the probe signals are repeated, and in the experimental case, the probe signal is slightly delayed in relation to the pump, which does not occur in the simulated case. This fact explains the slight delay between the quaternary signals obtained in the experimental results.

Fig. 11 shows the values of $r_{u p}, r_{i n t}$ and $r_{\text {low }}$ as a function of $r_{S}$, for $r_{G}$ varying from 3 to $7 \mathrm{~dB}$. These values were calculated using the sets of Eqs. (32) and (34), and the analytical, the simulated, and the experimental values were plotted in all of these cases, showing a rather good agreement for $r_{\text {up }}$ and $r_{\text {int }}$.

However, in the case of $r_{\text {low, }}$ a difference between the experimental and the analytical/simulated values for $r_{S}>6 \mathrm{~dB}$ appeared. Such difference increases when the value of $r_{G}$ increases. Moreover, the experimental values are always smaller than those of the simulated ones and the difference is always smaller than $1.4 \mathrm{~dB}$. This difference can be explained by the fact that when $r_{G}$ is significantly high, the quaternary signal ER, given by $r=r_{\text {low }} r_{\text {int }} r_{u p}=r_{G} r_{S}$ is also high. Thus, even when the measurements are taken with a fixed average power, the power of level " 00 " can reach a value around $-10 \mathrm{dBm}$; this value is comparable to the power level generated by the DSA photodiode dark current. Consequently, measurements of $r_{\text {low }}$ are not as accurate as those for smaller values of $r_{G}$.

To measure the $r_{S}$ values, the pump was simply turned off. Then, the ER of the binary signal at $\omega_{S}$ was measured from the eye-diagram at the DSA. A continuous-wave (cw) at $\omega_{S}$ and a modulated pump with ER $=r_{P}$ were used to obtain $r_{G}$. The PA gave two gains to the $c w$ 

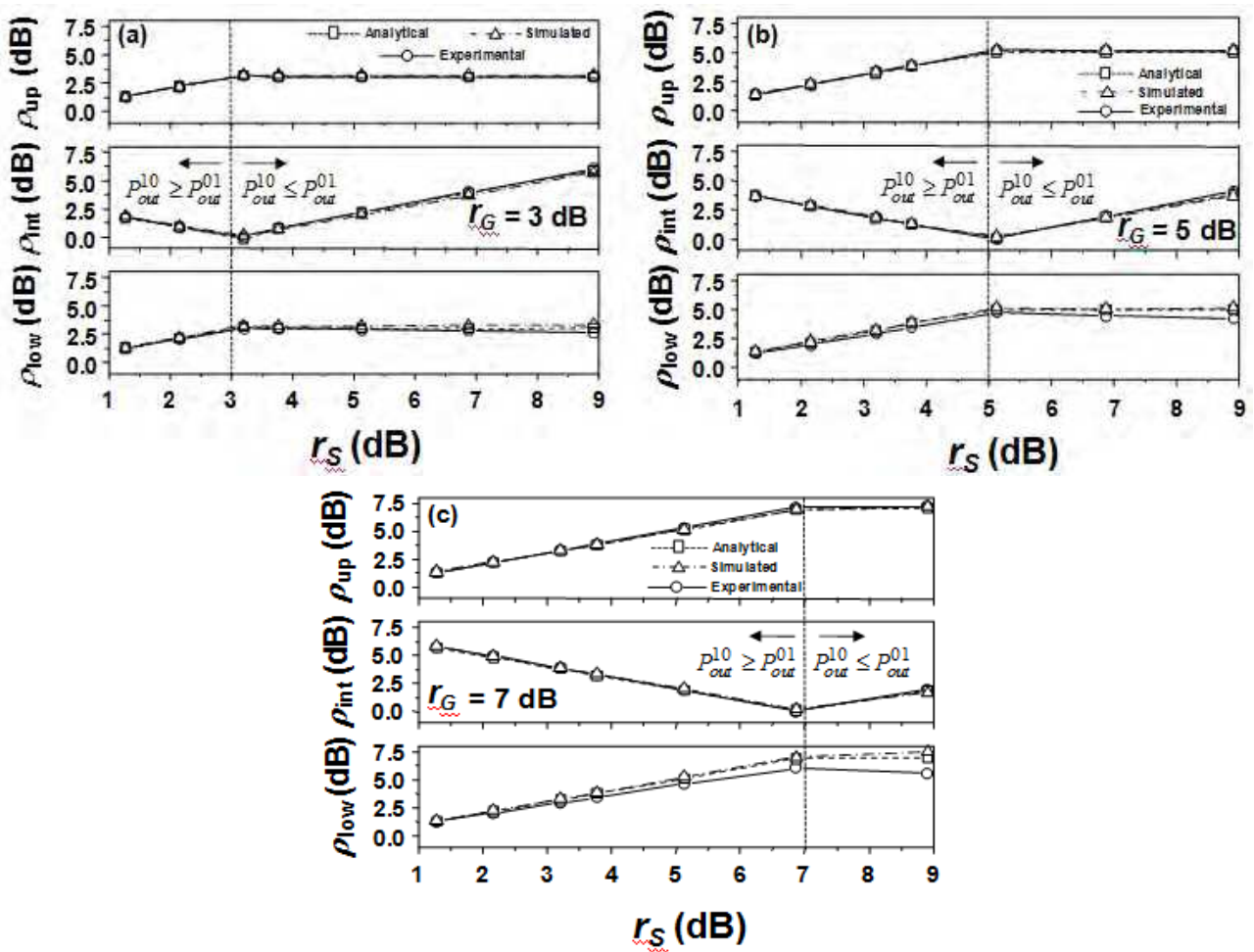

Fig. 11. Values of $r_{u p}, r_{i n t}$ and $r_{\text {low }}$ as a function of $r_{\mathrm{S}}$ for: (a) $r_{\mathrm{G}}=3.0 \mathrm{~dB},(\mathrm{~b}) r_{\mathrm{G}}=5.0 \mathrm{~dB},(\mathrm{c}) \mathrm{r}_{\mathrm{G}}=$ $7.0 \mathrm{~dB}$.

signal, generating a binary signal whose eye diagram can be seen on the DSA. The ER of such signal is precisely $r_{G}$.

Two sets of three ASK-4 eye-diagrams for the signal at frequency $\omega_{S}$ are exhibited in Figures 12 and 13 . The labels " 00 " and " 11 " used to identify the quaternary pattern levels always correspond to the lowest and uppermost levels, respectively. On the other hand, the labels " 01 " or " 10 " are always used to identify the two intermediate levels, but in this case the label of each specific level depends on the value of the signal extinction ratio $r_{S}$. For instance, for $r_{S}=2.2 \mathrm{~dB}$ (as in Fig. 12(a) the second and third power levels of the quaternary eye diagram correspond to labels " 01 " and " 10 ", respectively. This can be explained as follows. When $r_{S}=2.2 \mathrm{~dB}$ and the pump level is " 1 ", the parametric gain obtained by the signal level " 0 " is higher than that of the signal level " 1 " when the pump level is " 0 ".

Fig. 12(b) shows a particular case when the parametric gain given by the pump level " 1 " to the signal level " 0 " coincides with that given by the pump level " 0 " to probe level " 1 ". In such a situation, $r_{S} \approx r_{G}(3.8 \mathrm{~dB} \approx 4 \mathrm{~dB})$, and the quaternary signal degenerates into a ternary one. This situation must be avoided in practical applications. Fig. 12(c) presents the case $r_{S}=$ $6.8 \mathrm{~dB}>r_{G}$, which is the inverse case of the one shown in Fig. 12(a). Now, the power level of the label " 10 " is lower than that of the label " 01 ". This is because the gain of pump level " 1 " given to level " 0 " is higher than that given by the pump level " 0 " to signal level " 1 ". 

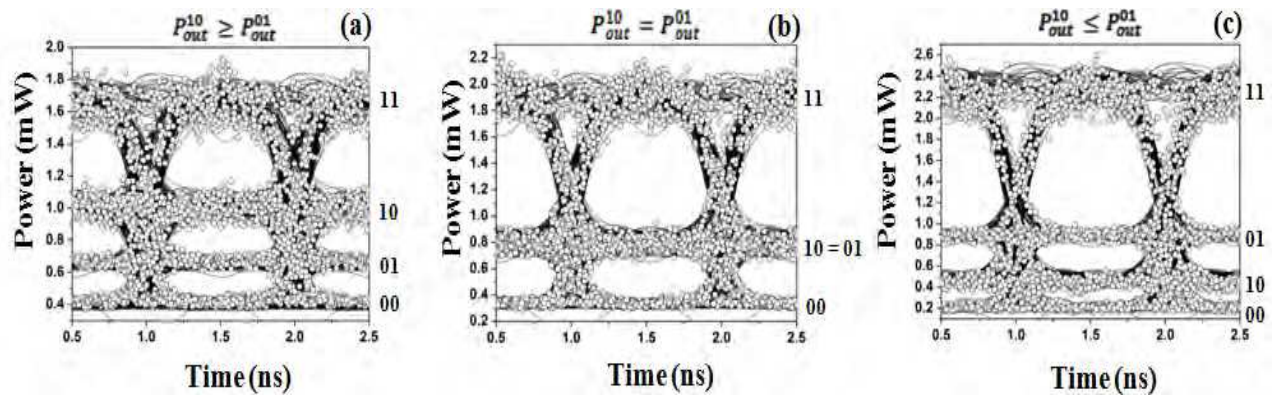

Fig. 12. Simulated (full lines) and experimental (white circles) eye diagrams for (a) $r_{\mathrm{S}}=2.2$ $\mathrm{dB},(\mathrm{b}) r_{\mathrm{S}}=3.8 \mathrm{~dB}$, and (c) $r_{\mathrm{S}}=6.9 \mathrm{~dB}$. In all the cases $r_{\mathrm{G}}=4 \mathrm{~dB}$.
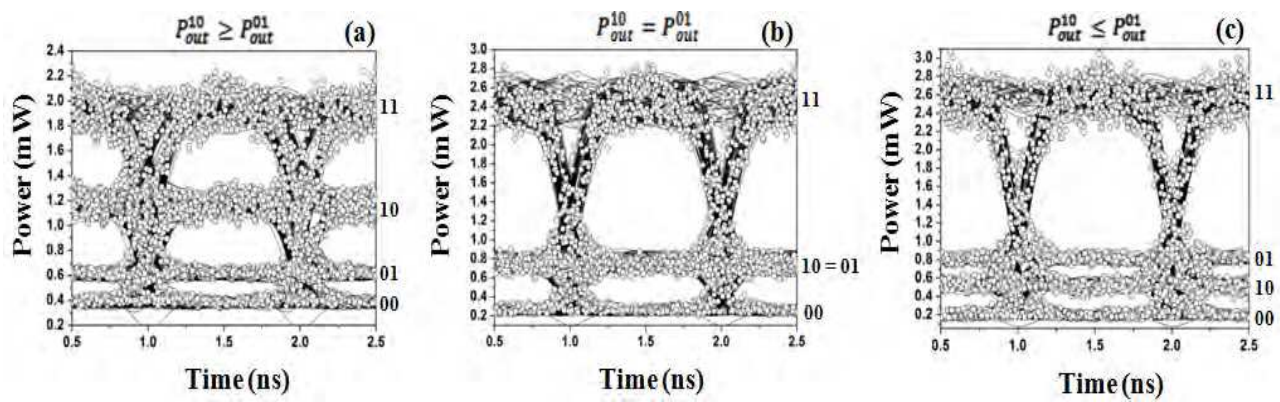

Fig. 13. Simulated (full lines) and experimental (white circles) eye diagrams for (a) $r_{S}=2.2$ $\mathrm{dB},(\mathrm{b}) r_{S}=5.1 \mathrm{~dB}$, and (c) $r_{S}=6.9 \mathrm{~dB}$. In all the cases $r_{G}=5 \mathrm{~dB}$.

A very good agreement between the simulated and experimental eye diagrams is observed in Figs. 12 and 13 for the two values of $r_{G}$ used, and similar results were observed for other values of $r_{G}$.

The eye diagrams for the quaternary signal that propagated through $75 \mathrm{~km}$ of standard fiber are shown in Fig. 14. The agreement between experimental eye diagrams and numerical results is also very good. The experimental BERs are lower than 10-13 and 10-11 before and after transmission, respectively. In principle, it is possible to improve such values by using a narrower optical band-pass filter or by increasing the quaternary signal average power. Table 1 presents estimations for the BERs before and after propagation by using Eqs. (29).

\begin{tabular}{|c|c|c|c|c|}
\hline & \multicolumn{2}{|c|}{ Before Propagation } & \multicolumn{2}{c|}{ After Propagation } \\
\hline & Experiment & Simulation & Experiment & Simulation \\
\hline$B E R_{\omega_{P}}$ & $1.1 \times 10^{-14}$ & $1.7 \times 10^{-14}$ & $7.4 \times 10^{-12}$ & $3.2 \times 10^{-12}$ \\
\hline$B E R_{\omega_{S}}$ & $8.0 \times 10^{-14}$ & $4.8 \times 10^{-14}$ & $7.6 \times 10^{-12}$ & $2.8 \times 10^{-11}$ \\
\hline
\end{tabular}

Table 1. BER estimation before and after fiber propagation. 

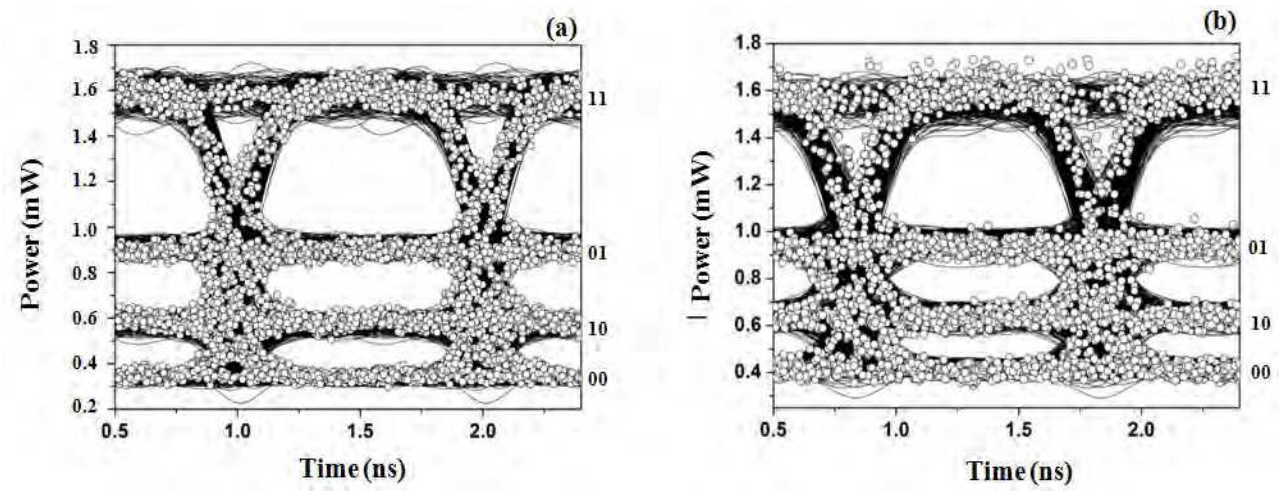

Fig. 14. Simulated (full lines) and experimental (white circles) eye diagrams: (a) before propagation, (b) after a $75 \mathrm{~km}$ standard fiber propagation. $r_{P}=0.86 \mathrm{~dB}\left(r_{G} \approx 2.5 \mathrm{~dB}\right), r_{S}=4.4$ $\mathrm{dB}$.

The recovery of the original binary information from the quaternary-amplitude signal can also be performed optically by using some known techniques (Oda \& Maruta, 2006), or by developing especial optical devices with the $S$ - and $U$-shaped transfer functions (Fagotto \& Abbade, 2010).

\section{All-optical generation of ternary amplitude-shift keying signals}

In the previous section we showed how to multiplex two 2-ASK signals into a single 4-ASK one. To perform such operation, it was necessary to provide a power offset to both input binary signals. As a result, the lowest level power of the of the 4-ASK signal was not null, which degrades the signal BER performance.

In this section, we discuss two other techniques that multiplex two binary signals and generate a 3-ASK signal rather than a 4-ASK one. The first one, OAM-3F, is based on FWM; it requires a power offset on just one of the input binary signals. The second, OAM-3P, utilizes PA and holds for two OOK input binary signals. For the same average power, ternary-amplitude signals present lower BERs than quaternary ones. Besides, the 3-ASK signal lowest power level is null for both techniques; this also contributes to reduce the BER of the multiplexed signal and it is an important advantage for the techniques presented here. However, recovering information relative to two binary signals from a single ternary one requires the use of some special signal characteristics. All of these aspects are detailed below.

\subsection{Optical amplitude multiplexing through fiber four-wave mixing}

\subsubsection{Theory}

The notation employed here is the same utilized in Section 3.1. The principle of OAM-3F is illustrated in Fig. 15a. Two co-polarized OOK signals at $\omega_{1}$ and $\omega_{2}$ are coupled and propagated through an optical fiber with nonlinear and dispersion parameters appropriate for favoring FWM; however, it is assumed that the signal at $\omega_{2}$ is an OOK, so $P_{2}^{0}(0)=0$. 

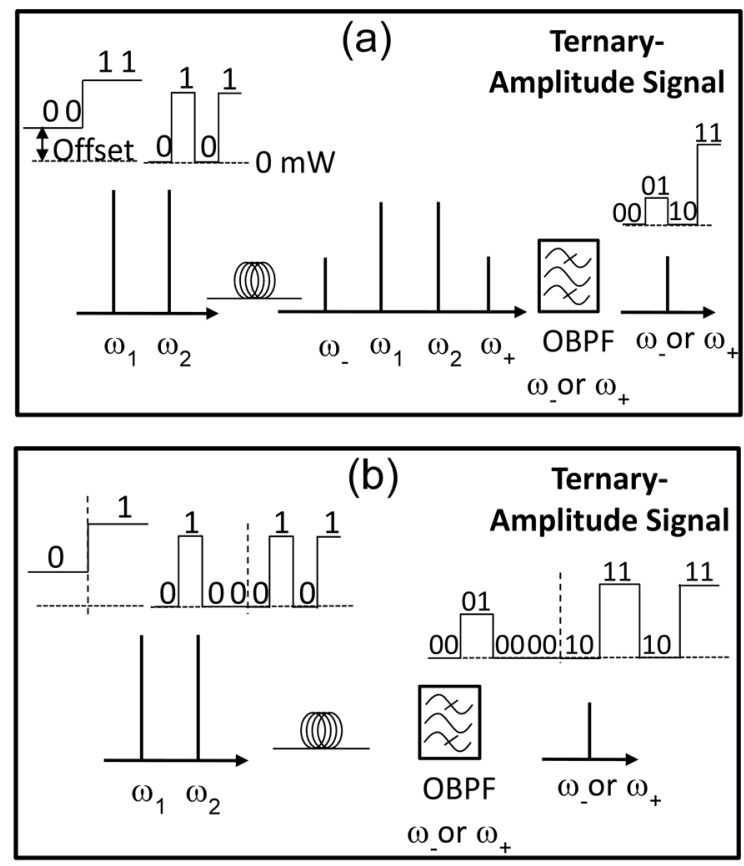

Fig. 15. Scheme illustrating the principle of operation of OAM-3F.

The OBPF is centered at $\omega_{+}$; therefore, when the signal at $\omega_{1}$ conveys a bit $i(=$ " 0 " or " 1 ") and the signal at $f_{2}$ carries a bit $j(=" 0$ " or " 1 "), the signal at the fiber output presents the following four power levels, $P_{\text {out }+}^{i j}$ :

$$
\begin{gathered}
P_{\text {out }+}^{00}=k_{+} P_{1}^{0}(0)\left[P_{2}^{0}(0)\right]^{2}=0 \\
P_{\text {out }+}^{10}=k_{+} P_{1}^{1}(0)\left[P_{2}^{0}(0)\right]^{2}=0 \\
P_{\text {out }+}^{01}=k_{+} P_{1}^{0}(0)\left[P_{2}^{1}(0)\right]^{2} \\
P_{\text {out }+}^{11}=k_{+} P_{1}^{1}(0)\left[P_{2}^{1}(0)\right]^{2}
\end{gathered}
$$

Since $P_{\text {out }+}^{00}=P_{\text {out }+}^{10}=0$, the output signal now is a 3 -ASK one. Recovering information transmitted by the $\omega_{2}$ signal from the generated ternary amplitude signal is a straightforward task. If one detects power levels corrspeonding to $P_{\text {out }+}^{01}$ or $P_{\text {out }+}^{11}$, the signal at $f_{2}$ clearly transmits a bit " 1 "; otherwise it transmits a bit " 0 ". This may be promptly verified by inspecting Fig. 15a or Eqs. (35).

On the other hand, recovering the pump information from the generated ternary amplitude signal is, in principle, not possible because $P_{\text {out }+}^{00}=P_{\text {out }+}^{10}$. This power level ambiguity implies 
that when the lowest (null) power level is detected, the signal at $f_{1}$ may either convey a " 0 " or a " 1 "-bit. However, it is possible to solve this problem if the following assumptions are made:

a. The line code used by the signal at $\omega_{2}$ guarantees a maximum of $(N-1)$ bits 0 in a row, i.e. in a sequence of $N$ bits there is at least one bit " 1 ". In fact, this characteristic is implemented by most practical line codes to minimize the chance of the receiver loosing synchronism to the transmitter. For example, the line code utilized in Gigabit Ethernet standard, called $8 \mathrm{~B} / 10 \mathrm{~B}$, assures that no bit sequence presents more than four bits " 0 " in a row of ten bits;

b. the signal bit rate at $\omega_{2}, R_{b 2}$, is higher than the signal bit rate at $\omega_{1}, R_{b 1}$ :

$$
R_{b 2}=N R_{b 1}
$$

where $N$ is the same integer number considered in assumption (a), and

c. to recover information conveyed by the signal at $\omega_{1}$, such a signal is oversampled at a rate $R_{b 2}$. Because of (b), this oversampling process establishes that $N$ samples of the signal at $\omega_{1}$ are obtained for each of its bits. However, following (a), at least in one of these samples, the signal at $\omega_{2}$ shall transmit a " 1 "-bit. Therefore, the detection rules for the signal at $\omega_{1}$ are the following. In a sequence of $N$ samples:

i. if only power levels $P_{\text {out }+}^{11}$ and $P_{\text {out }+}^{00}$ are detected, then the signal at $\omega_{1}$ sent a "1-bit";

ii. if only power levels $P_{\text {out }+}^{01}$ and $P_{\text {out }+}^{00}$ are detected, then the signal at $\omega_{1}$ sent a " 0 -bit";

iii. if any other combination of power levels is detected, then there is an error and information should be discarded.

The detection rules just described may be verified by simple inspection of Fig. 15b, where rules (a), (b), and (c) were considered for $\mathrm{N}=4$.

As stated before, power level optimization for optical multi-amplitude signals depends on the kind of the dominant noise and it is analyzed in (Walklin \& Conradi, 1999). In case ASE noise is dominant, such optimal distribution must follow a quadratic law; for a ternaryamplitude signal, this means that power levels must be distributed following proportions of 0: 1: 4. Eqs. (35d) show that such distribution may be easily obtained by setting:

$$
\frac{P_{\text {out }+}^{11}}{P_{\text {out }+}^{01}}=\frac{P_{1}^{1}(0)}{P_{1}^{0}(0)}=r_{1}=4
$$

Therefore, power level optmization may be easily achieved when using this technique. In fact, OAM-3F is able to generate 3-ASK signals with arbitrary power level distributions, by solely setting the ER of the signal at $\omega_{1}$.

After inspecting Fig. 15b and following a reasoning similar to the one considered in Section 3.1 , it is easy to conclude that:

$$
B E R_{2}=Q\left(\frac{i_{01}-i_{00}}{\sigma_{01}+\sigma_{00}}\right)
$$


To estimate $B E R_{1}$, we assume that an EDC, such as the one illustrated in Fig. 2, is programmed to decide which bit was sent by the signal at $\omega_{1}$ based only on the first non-null sample of the acquired $N$ samples (assumption (c) above). In this case, the EDC interprets that the signal $\omega_{1}$ sent a ' 0 '-bit or ' 1 '-bit when, respectively, the intermediate or the highest power level is detected. In this case, one can write:

$$
\begin{aligned}
B E R_{1} & =P\left(\varepsilon \mid l^{\prime} l^{\prime}\right) P\left(' l^{\prime}\right)+P\left(\varepsilon \mid i^{\prime}\right) P\left(i^{\prime}\right)+P\left(\varepsilon||^{\prime} h\right) P\left({ }^{\prime} h '\right) \\
& =\frac{1}{2} Q\left(\frac{i_{01}-i_{10}}{\sigma_{01}+\sigma_{10}}\right) \cdot \frac{1}{2}+\left[Q\left(\frac{i_{01}-i_{10}}{\sigma_{01}+\sigma_{10}}\right)+Q\left(\frac{i_{11}-i_{01}}{\sigma_{11}+\sigma_{01}}\right)\right] \cdot \frac{1}{4}+Q\left(\frac{i_{11}-i_{01}}{\sigma_{11}+\sigma_{01}}\right) \cdot \frac{1}{4} \\
& =\frac{1}{2}\left[Q\left(\frac{i_{01}-i_{10}}{\sigma_{01}+\sigma_{10}}\right)+Q\left(\frac{i_{11}-i_{01}}{\sigma_{11}+\sigma_{01}}\right)\right]
\end{aligned}
$$

where ' $l$ ', ' $i$ ', and ' $h$ ' stand, respectively, for the lowest, intermediate, and highest amplitude levels, $P(x)$ is the probability of occurrence of level $x\left(x={ }^{\prime} l^{\prime},{ }^{\prime}{ }^{\prime}\right.$ ', and ' $\left.h{ }^{\prime}\right)$, and $P(\varepsilon \mid x)$ is the conditional probabylity that an error occurs given that level $x\left(x={ }^{\prime} l l^{\prime},{ }^{\prime}{ }^{\prime}\right.$ ', and ' $h$ ') is detected.

Although the theory presented above was derived for a signal with finite ER at $\omega_{1}$ and an OOK signal at $\omega_{2}$, the proposed technique could also be applied for an OOK signal at $\omega_{1}$ and a signal with finite ER at $\omega_{2}$. Also, the FWM component selected by the OBPF could be at $\omega$. Eqs. (35)-(38) should be adapted in all of the possible combinations. Nevertheless, the general aspects treated here, like the power level optimization would still hold.

\subsubsection{Results and discussion}

Considering the system shown in Fig. 15b, Fig. 16 presents simulation results for bit sequences for the (a) signal at $\omega_{1}=193.1 \mathrm{THz}$ with $r_{1}=2$ and $R_{b 1}=2.5 \mathrm{~Gb} / \mathrm{s}$, (b) signal at $\omega_{2}=$ $193.2 \mathrm{THz}$ with $R_{b 2}=10 \mathrm{~Gb} / \mathrm{s}$, and (c) signal at the OBPF output at $\omega_{+}=193.3 \mathrm{THz}$. Fiber parameters are: $\lambda_{0}=1552.52 \mathrm{~nm}, S_{0}=0.017 \mathrm{ps} / \mathrm{nm}^{2} / \mathrm{km}, \gamma=5.3 \mathrm{~W}^{-1} \mathrm{~km}^{-1}, \alpha=0.2 \mathrm{~dB} / \mathrm{km}$, and $L=3.0 \mathrm{~km}$. The signal observed in Fig. 16c clearly represents a ternary-amplitude one. Moreover, the same power levels are obtained when the bits of the pump and the probe signals are repeated.

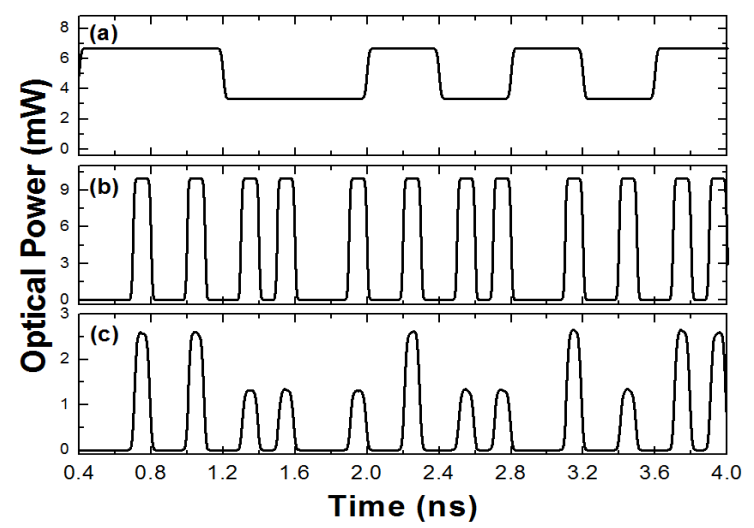

Fig. 16. Input signals at (a) $\omega_{1}$ and (b) $\omega_{2}$ and output signal at (c) $\omega_{+}$. 
Fig. 17 plots the eye diagrams for the ternary-amplitude signals with average power of 1 $\mathrm{mW}$, for $r_{1}=$ (a) 2.0, (b) 4.0, and (c) 6.0. Eye-diagrams present $P_{\text {out }+}^{11} / P_{\text {out }+}^{01}$, respectively, of 1.9, 3.9, and 5.8, which are in good agreement with the values predicted by the theory previously presented. In these simulations, ASE noise was deliberately added to the signal in order to provide a typical OSNR of $65 \mathrm{~dB}$ at the receiver. In the three presented cases,
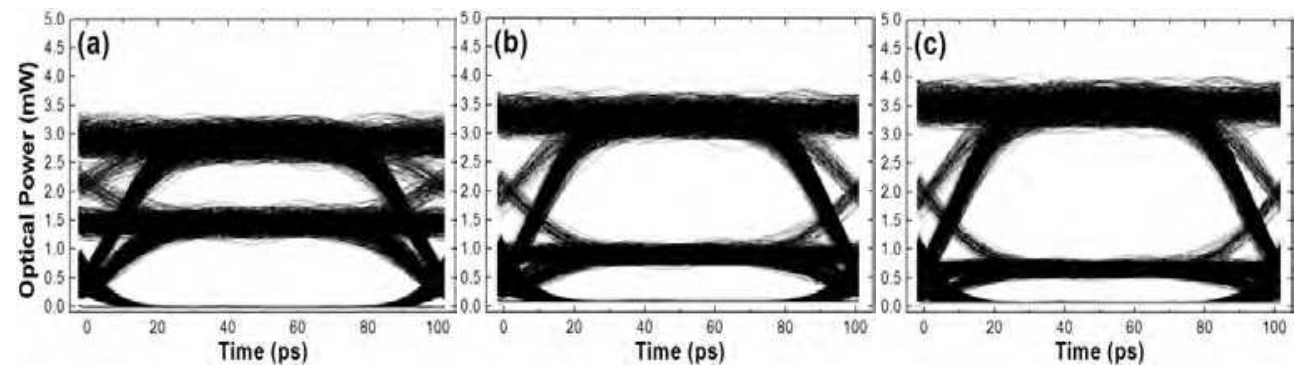

Fig. 17. Eye diagrams for $r_{1}=$ (a) 2, (b) 4 , and (c) 5 .

$B E R_{2}$ is inferior to $10^{-15}$, which may be considered as error-free. $B E R_{1}$ achieves a maximum of 4.6 10-12 for $r_{1}=2.0$. Such low BER values suggest OAM-3F viability in practical situations. The uppermost amplitude level is the noisier one; this is a consequence of FWM combining the noise from two "1"-bits of the input binary signals, which are more affected by ASE noise than the "0"-bits.

\subsection{Optical amplitude multiplexing through fiber parametric amplification}

\subsubsection{Theory}

Here, we use the same notation as in Section 3.2. Fig. 18 illustrates a diagram for implementing OAM-3P. Two co-polarized OOK signals at optical carriers $\omega_{P}$ and $\omega_{S}$ are coupled and co-propagated through an optical fiber that provides the appropriate dispersion regime and nonlinear parameter to support parametric amplification. However, both input signals are OOK; hence, $P_{P}^{0}(0)=P_{S}^{0}(0)=0$. It is assumed that $P_{P}^{1}(0)>>P_{S}^{1}(0)$. In this case, three power levels are possible for the signal at $\omega_{P}$ at the OBPF output. The first one occurs when both signals transmits a "1"-bit and is given by:

$$
P_{\text {out }}^{11}=G_{P}^{1} P_{S}^{1}(0)
$$

Obviously, this corresponds to the situation where the probe signal is amplified by the pump. In the second case, the probe signal transmits a " 1 "-bit whereas the pump transmits a " 0 "-bit. In this situation, the probe signal is not amplified by the pump and, assuming low fiber dispersion, it is solely attenuated by the fiber. In this situation, the power at the OBPF output is:

$$
P_{\text {out }}^{01}=P_{S}^{1}(0) e^{-\alpha L}
$$




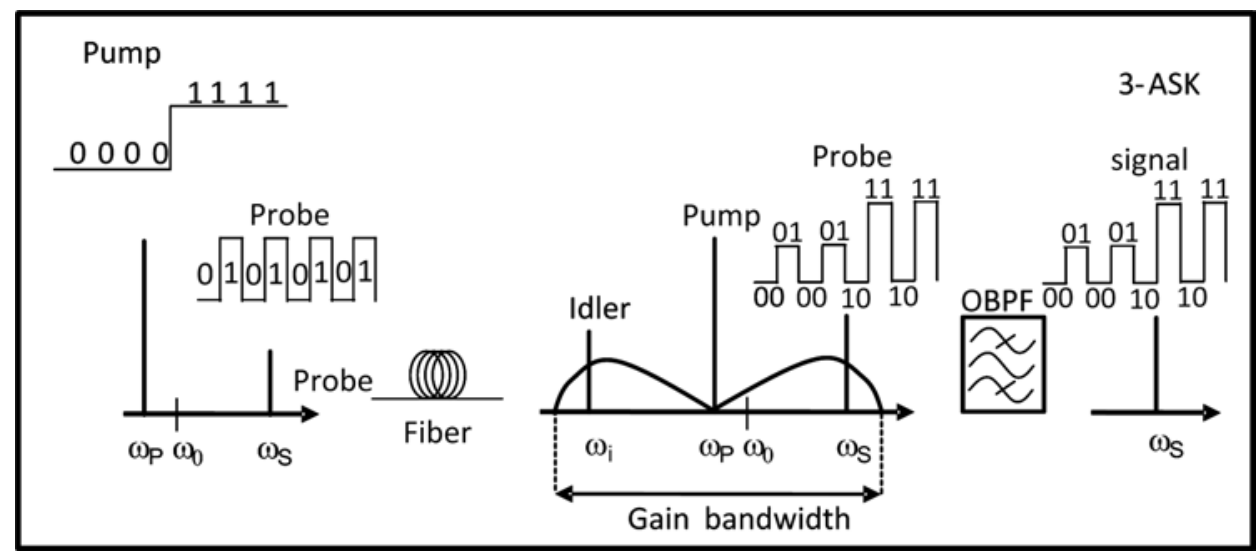

Fig. 18. Scheme for 3-ASK generation $\left(\omega_{k}\right.$ with $k=P, S, i$ representing the angular frequencies of the pump, the signal and the idler, and $\omega_{0}=2 \pi \mathrm{c} / \lambda_{0}$, where $\lambda_{0}$ is the zero dispersion wavelength of the fiber).

Finally, the third case corresponds to the one where the probe signal transmits a " 0 "-bit. Then, no matter the bit set by the pump, the power at the OBPF output will be null:

$$
P_{\text {out }}^{00}=P_{\text {out }}^{10}=0
$$

We have already treated a situation where information from two input binary signals need to be recovered from a ternary-amplitude one in the previous sub-section. In fact, here we may apply the same reasoning used in Section 4.1. Particularly, the detection rule for recovering information of the signal at $\omega_{S}$ from the photo-detected ternary-amplitude signal is the same considered for the signal at $\omega_{2}$ in Section 4.1. So, it is interpreted that the signal at $\omega_{S}$ transmits a bit " 1 " if the power levels corresponding to $P_{\text {out }}^{01}$ or $P_{\text {out }}^{11}$ are detected and that it transmits a bit " 0 " if a null power is received.

Because of the ambiguity expressed by (39c), recovering the signal information at $\omega_{P}$ from the photodetected ternary-amplitude requires the use of assumptions equivalent to the ones utilized in Section 4.1:

a. the line code used by the signal at $\omega_{s}$ guarantees a sequence of $N$ bits contains at least one bit " 1 ".

b. the bit rate of the signal at $\omega_{S}, R_{b S}$, is related to the bit rate of the signal at $\omega_{P}, R_{b P}$ by:

$$
R_{b S}=N R_{b P},
$$

c. the signal at $\omega_{P}$ is oversampled at a rate $R_{b S}$ and the following detection rules are used:

i. if only power levels $P_{\text {out }}^{11}$ and $P_{\text {out }}^{00}$ are detected, then the signal at $\omega_{P}$ sent a "1-bit";

ii. if only power levels $P_{\text {out }}^{11}$ and $P_{\text {out }}^{00}$ are detected, then the signal at $\omega_{P}$ sent a "0-bit";

iii. if any other combination of power levels is detected, then there is an error and information should be discarded. 
Due to the similarities between the ternary-amplitude signals generated by OAM-3F and OAM-3P, it is also possible to write the bit error rates associated to the signals at $\omega_{S}$ and $\omega_{P}$, respectively, as:

$$
\begin{gathered}
B E R_{S}=Q\left(\frac{i_{01}-i_{00}}{\sigma_{01}+\sigma_{00}}\right) \\
B E R_{P}=\frac{1}{2}\left[Q\left(\frac{i_{01}-i_{10}}{\sigma_{01}+\sigma_{10}}\right)+Q\left(\frac{i_{11}-i_{01}}{\sigma_{11}+\sigma_{01}}\right)\right]
\end{gathered}
$$

It should be observed that, as OAM-3S, OAM-3P also offers arbitrary power level distribution. In fact, since $P_{\text {out }}^{00}=0$ the ratio between the two other power levels may be regulated by simply setting $G_{P}^{1}$. This may be easily verified by dividing (55a) by (55b):

$$
\frac{P_{o u t}^{11}}{P_{\text {out }}^{01}}=G_{P}^{1} e^{-\alpha L}
$$

Thanks to this characteristic, power level distribution of ternary-amplitude signals generated by OAM-3P may be set to minimize BER under the dominance of any kind of noise.

\subsubsection{Results and discussion}

Fig. 19 illustrates an experimental setup utilized for generating 3-ASK signals through OAM-3P with $\mathrm{N}=4$. Pump consists of a modulated signal at $f_{P}=192.3 \mathrm{THz}$. It is phasemodulated by radio-frequencies of 601 and $983 \mathrm{MHz}$ to prevent the deletrious effects of Brillouin backscattering. This pump is further amplified and filtered in such a way that its power at the fiber input can be varied from 9.3 to $10.8 \mathrm{dBm}$. A 215-1 pseudo-random bit sequence (PRBS) externally modulates a $c w$ at $f_{S}=193.2$ with $R_{b S}=1.0 \mathrm{~Gb} / \mathrm{s}$. Its power at the fiber output is of $-19 \mathrm{dBm}$. The highly nonlinear dispersion-shifted fiber (HNL-DSF) is characterized by $\lambda_{0}=1555.4 \mathrm{~nm}, \Delta \lambda_{0}=10 \mathrm{~nm}, S_{0}=0.017 \mathrm{ps} / \mathrm{nm}^{2} / \mathrm{km}, \gamma=9.1 \mathrm{~W}^{-1} \mathrm{~km}^{-1}, \alpha=$ $0.83 \mathrm{~dB} / \mathrm{km}$, and L=3.0 km. Signals at fiber output are filtered, amplified and filtered again. Both filters are centered at $f_{S}$. The 3-ASK signal at $f_{S}$ is then either analyzed by a DSA or transmitted through a 40-km long SMF link in KyaTera Network (FAPESP, (n.d.)).

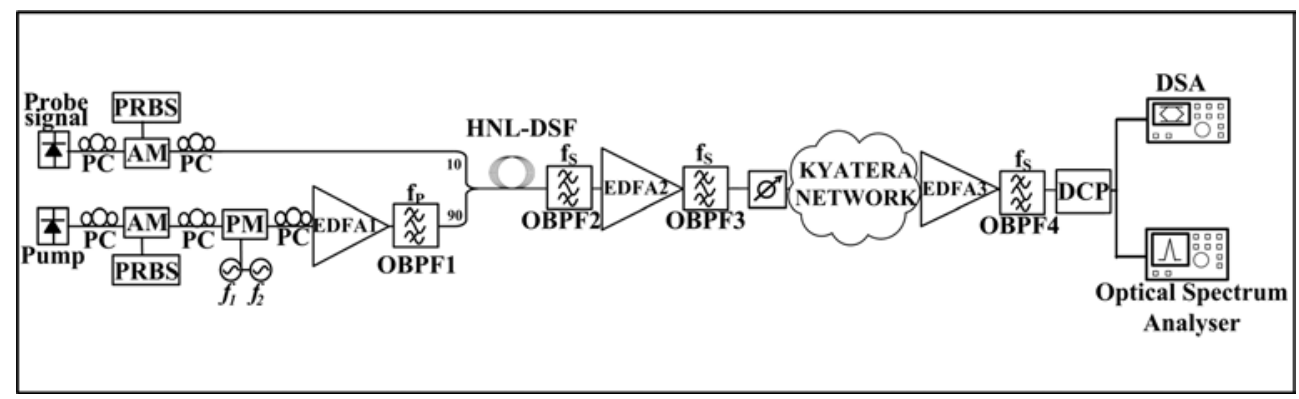

Fig. 19. Experimental setup. 
In this second situation, the signal is further amplified, filtered and goes through a dynamic polarization controller (DPC) before being analyzed by the DSA. The DPC ensures the output signal state of polarization (SOP) will be the same, no matter its input SOP. This is important because the utilized link uses aerial cables that frequently introduces SOP changes due to environmental condition variations. Signal spectra at the (a) HNL-DSF output and (b) at the OBPF3 output, for an OSNR of $\sim 31 \mathrm{~dB}$, are shown in Fig. 20.

Fig. 21 plots bit sequences for the signals at (a) $f_{P}$ and (b) $f_{S}$, and (c) for the correspondent generated OAM-3P. It is clearly verified that the signal in Fig. 21c represents a ternaryamplitude one. Moreover, as with the other presented techniques, the same power levels are obtained when the bits of the input binary signals are repeated.
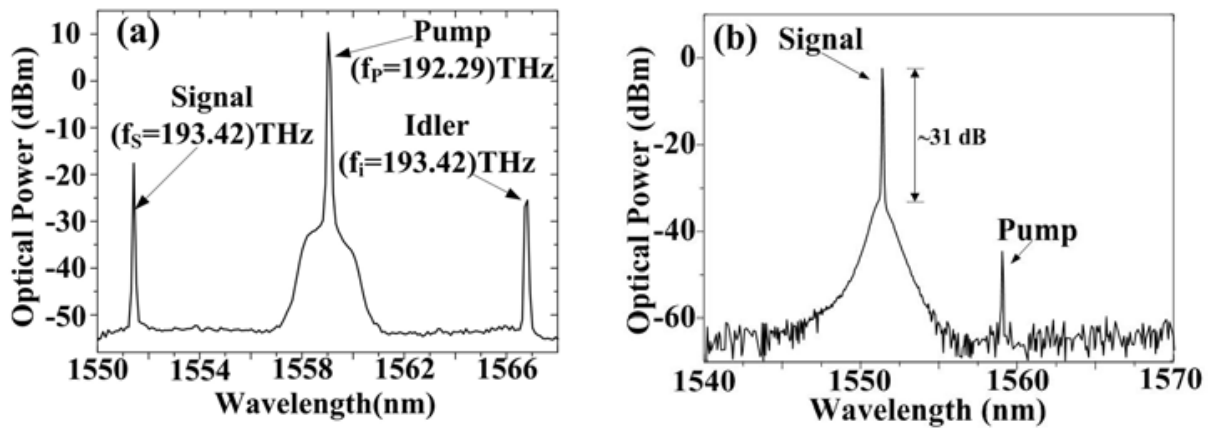

Fig. 20. Power spectra at the (a) HNL-DSF output and (b) OBPF3 output.

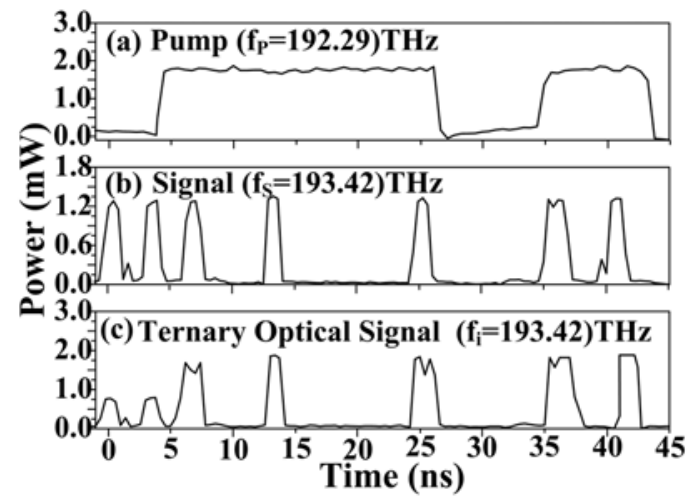

Fig. 21. Binary sequences (a) Pump (b) Signal (c) Ternary Amplitude Optical Signal for r=2.5.

Finally, Fig. 22 presents eye-diagrams for ternary-amplitude signals before and after network propagation with $P_{\text {out }}^{11} / P_{\text {out }}^{01}=3.0,3.5$, and 4.0 . Such values were obtained by varying the pump power, i.e., by controlling the parametric gain provided by the modulated pump. Using (41) we find that the lowest BERs are obtained for $P_{\text {out }}^{11} / P_{\text {out }}^{01}=2.0$ and $B E R_{S}=$ $3.510^{-15}$ and $B E R_{P}=4.710^{-14}$. These low values, show that the technique may be properly applied to practical applications. 

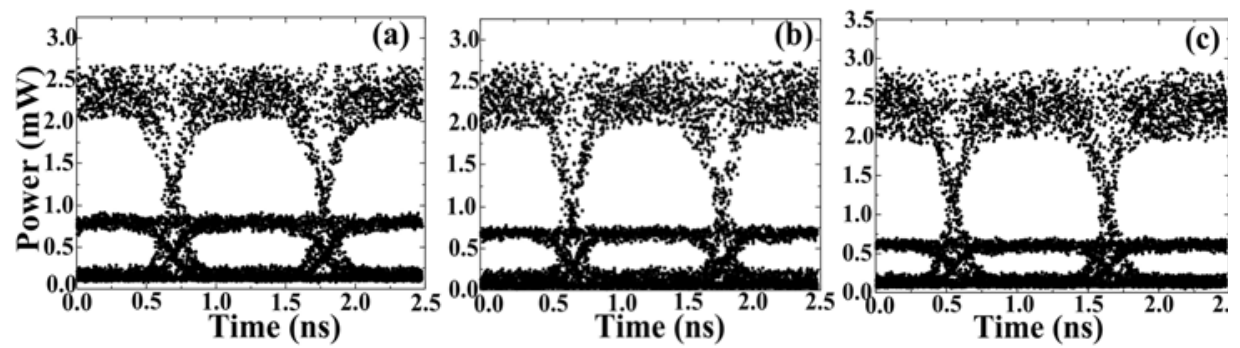

Fig. 22. Eye diagrams of ternary-amplitude signals after $40 \mathrm{~km}$ for $P_{\text {out }}^{11} / P_{\text {out }}^{01}=(\mathrm{a}) 3.0$, (b) 3.5, and (c) 4.0.

\section{Applications and comparisons}

In Sections 3 and 4 we have presented four techniques that convert two input binary signals into either a 4-ASK or a 3-ASK one. Due to the fast response of parametric interaction in fibers, all of these techniques are bit-rate independet for practical bit rates. Futhermore, all of them are able to transmit information from both input signals simultaneously and in the same bandwidth. Therefore, they could be used, for instance, to multiplex data from two different wavelengths into a single one, which results in bandwidth savings (Abbade et al., 2005).

Another possible application is to use one of the input signals to introduce a label to the second one. This optical labeling operation is illustrated in Fig. 23 and would be useful when binary data is entering the domain of an optical packet switching network (OPSN) and needs to be converted to an optical packet (Abbade et al., 2006a; Abbade et al., 2010a).

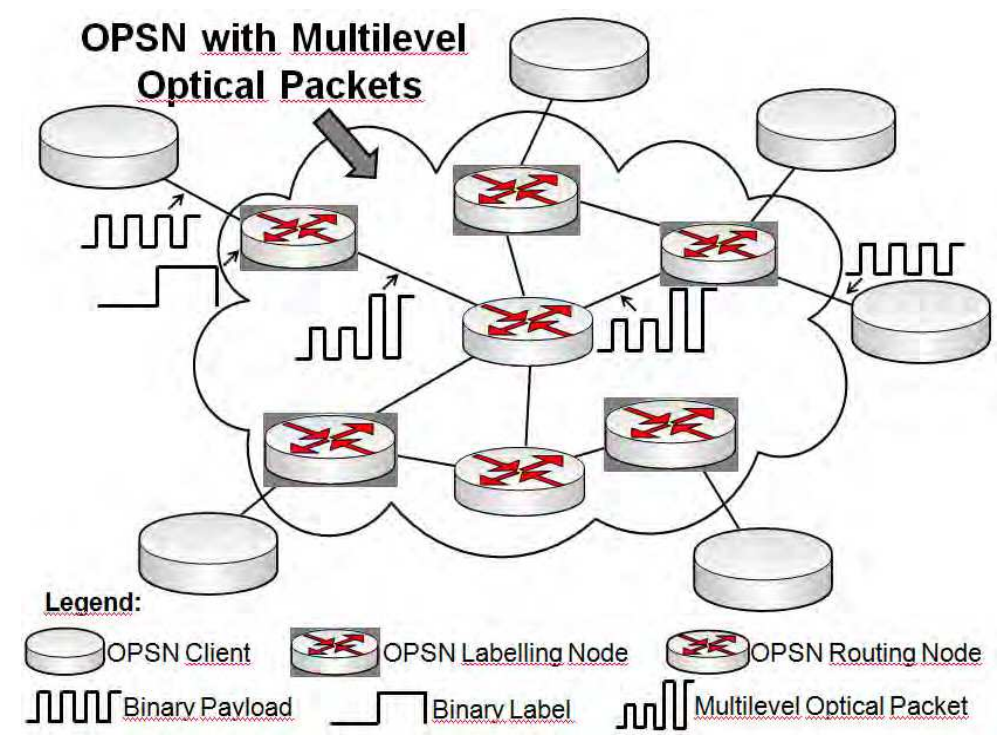

Fig. 23. Optical labeling application 
Yet another possible application is to use OAM-4F and OAM-4P in several stages in a way that binary signals are successively converted into an analog signal (Abbade et al., 2010a). For example, OAM- $4 \mathrm{~F}$ could be used to multiplex two binary signals into a quaternary one, which could be combined to another binary signal into an octary one and so on, until the number of levels is high enough to make the signal resemble an analog one.

In spite of being employed to the same applications and utilizing very similar setups, there are marked differences among the analyzed techniques. Some of them have already been mentioned; here, it is interesting to summarize them and present some further distinctions.

One of these is that two of the considered techniques, OAM-4F and OAM-4P, generate 4ASK signals and the other two, OAM-3F and OAM-3P, produce 3-ASK signals. Both of these modulation formats are advantageous over OOK modulation because they provide higher tolerance to chromatic dispersion degradations. The considered 3-ASK signals present lower BERs than the 4-ASK not only because their number of levels is smaller, but also because their lowest level has null power. In fact, the power level distribution of these 3-ASK signals may be arbitrarily set, which does not happen for the considered 4-ASK signals. OAM-3P still has the advantage of not needing any power offset for its input binary signals, i.e., it can operate with conventional OOK input signals.

On the other hand, OAM-3F and OAM-3P only work under some special conditions, which comprises restrictions to the input signals line codes and bit rates and also demands oversampling the output signal to recover information transmitted by the pump signal. This makes OAM-3F and OAM-3P more suitable for the optical labeling application, where label bit rate is typically inferior to that of payload (otherwise, packet overhead could be very high). By their turn, OAM-4F and OAM-4P are independent of the utilized line codes and bit rates.

Since the techniques based on FWM, OAM-4F and OAM-3F, do not need a pump to transfer power to another signal, they may be accomplished by utilizing lower powers than OAM-4P and OAM-3P require. In fact, the average power of the input signals in experiments of OAM- $4 \mathrm{~F}$ were around $\sim 12 \mathrm{dBm}$ (Abbade et al., 2006b), whereas for the OAM-4P setup an average pump power as high as $20 \mathrm{dBm}$ was necessary (Abbade et al., $2010 \mathrm{~b}$ ). With such high powers, Brillouin backscattering becomes relevant; thus, the experimental setup of OAM-4P and OAM-3P needs some additional equipment to reduce the influence of this effect and becomes more complex than the setup utilized by the other two techniques. In particular, if phase modulators and RF generators are used to prevent Brillouin backscattering, then phase noise may cause further degradations to the generated signal. However, in spite of these drawbacks and in opposition to FWM, PA does not broaden the linewidth of the generated signal. Therefore, if no measures are taken to compensate dispersion, the multi-amplitude signals generated by OAM-4P and OAM-3P can propagate through longer distances than the ones produced by OAM- $4 \mathrm{~F}$ and OAM-3F.

Another important advantage for OAM-4P and OAM-3P techniques is that the generated multi-amplitude signal is at the same wavelength as one of its inputs. This does not occur with OAM-4F and OAM-3F strategies, where it is necessary to know the spacing between the input signals frequencies to set the filter that will select the output signal. Table 2 summarizes the most important differences commented above. 


\begin{tabular}{|l|c|c|c|c|c|c|c|}
\hline Technique & $\begin{array}{c}\text { One of the } \\
\text { Input } \\
\text { Channels need } \\
\text { a higher bit- } \\
\text { rate }\end{array}$ & $\begin{array}{c}\text { One of the } \\
\text { Input } \\
\text { Channels need } \\
\text { a higher } \\
\text { power }\end{array}$ & $\begin{array}{c}\text { Number of } \\
\text { Input } \\
\text { Channels } \\
\text { with power } \\
\text { off-set }\end{array}$ & $\begin{array}{c}\text { Output at a } \\
\text { new } \\
\text { wavelength }\end{array}$ & $\begin{array}{c}\text { Output } \\
\text { with } \\
\text { broadened } \\
\text { linewidth }\end{array}$ & $\begin{array}{c}\text { Output Signal } \\
\text { with Arbitrary } \\
\text { Power Level } \\
\text { Distribution }\end{array}$ & $\begin{array}{c}\text { Output } \\
\text { Signal } \\
\text { BER }\end{array}$ \\
\hline OAM-4F & No & No & 2 & $Y e s$ & Yes & No & High \\
\hline OAM-4P & No & Yes & 1 or 2 & No & No & No & High \\
\hline OAM-3F & Yes & No & 1 & $Y e s$ & Yes & Yes & Low \\
\hline OAM-3P & Yes & Yes & 0 & No & No & Yes & Low \\
\hline
\end{tabular}

Table 2. Comparison among the presented techniques.

\section{Conclusion}

The utility of optical fibers goes far beyond simple transmission in Optical Communication systems. In this work we have considered fiber applications to the very promising field of all-optical digital signal processing. Particularly, we focused on four techniques that allow the conversion of input binary signals into ternary- or quaternary-amplitude ones. Three of these techniques had already been analyzed in other reports; OAM-3F was, however, proposed and analyzed here for the first time.

Although we considered solely the generation of multi-amplitude signals, some modifications on the presented techniques could allow for optical phase multiplexing, as well. For example, in (Zhou et al., 2006) OAM-4F was adapted to merge two differential phase-shift keying signals into a single differential quadrature phase-shift keying one. Finally, it should be noted that the techniques discussed here could, in principle, be extended to any other nonlinear material that supports FWM and PA, such as semiconductor optical amplifiers and silicon chips. The latter is of special importance because it would allow the aforementioned applications to be performed in chip-to-chip communications, in the emerging field of silicon photonics.

\section{Acknowledgment}

This work was supported by FAPESP grant 08/57857-2 and CNPq grants 574017/2008-9 and 309031/2008-7. Authors thank Dr. Hugo L. Fragnito for invaluable discussions. Authors also thank VPIPhotonics Inc. for providing academic licenses of VPITransmissionMaker.

\section{References}

Abbade, M.L.F.; Fagotto, E.A.M.; Braga, R. S.; Fonseca, I. E.; Moschim, E. \& Barbosa, F.R. (2005). Optical Amplitude Multiplexing Through Four-Wave Mixing in Optical Fibers. IEEE Photonics Technology Letters, vol. 17, no. 1, pp. 151 - 153.

Abbade, M.L.F.; Almeida, F.P.; Branquinho, F. G. G.; Fagotto; Braga, R.S.; Callegari, F.A.; Rocha, M.L.; Rossi, S.M.; Boggio, J.M.C.; Fragnito, H.L. (2006a). Optically Generated Quaternary Packets: Transmission over the KyaTera Network. Proceedings of the 19th Annual Meeting of the IEEE, Lasers and Electro-Optics Society, Montreal, Canada, p. 557-558, 2006.

Abbade, M.L.F.; Fagotto, E.A.M.; Braga, R.S.; Barbosa, F.R.; Moschim, E. \& Fonseca, I. E. (2006b). Quaternary Optical Packets Generated by Fiber Four-Wave Mixing. IEEE Photonics Technology Letters, vol. 18, no. 2, pp. 331 - 333. 
Abbade, M.L.F.; Fagotto, E.A.M.; Braga, R.S.; Moschim, E.; Fonseca, I.E. \& Callegari, F.A. (2006c). All-optical generation of quaternary amplitude signals. IEE Electronics Letters, vol. 42, no. 24.

Abbade, M.L.F.; Costa, A.L.A.; Barbosa, F.R.; Durand F.R.; Marconi, J.D. \& Moschim E. (2010a). Optical Amplitude Multiplexing through Parametric Amplification in Optical Fibers. Optics Communications, v. 283, p. 454-463.

Abbade, M.L.F.; Marconi, J.D.; Costa, A.L.A.; Barbosa, F.R. ;Moschim, E. \& Fragnito, H. L. (2010b). All-optical Generation of Quaternary Amplitude-Shift Keying Signals through Parametric Amplification. Proceedings of the 12th International Conference on Transparent Optical Networks (ICTON),Munich, Germany, June, 2010.

Abbade, M.L.F.; Costa, A.L.A., Marconi, J.D.; Cardoso, V.V., Fragnito, H.L. \& Moschim, E. (2011). Optical Labelling through Parametric Amplification. Proceedings of the 13th International Conference on Transparent Optical Networks (ICTON), Stockholm, Sweden, June, 2011.

Agrawal, G.P. (2001). Nonlinear Fiber Optics, 3rd ed., New York: Academic Press.

Boyd, R.W. (2008). Nonlinear Optics, 3rd ed., Academic Press.

Brzozowski, L. \& Sargent, E.H. (2001). All-optical analog-to-digital converters, hardlimiters, and logic gates. Journal of Lightwave Technology, vol. 19, no.1, pp. 114-119.

Buck, J.A. (2005). Fundamentals of Optical Fibers, John Wiley \& Sons, Inc..

Butcher, P.N. \& Cotter, D. (1990). The Elements of Nonlinear Optics, Cambridge Univ. Press, Cambridge, England UK.

Chavez Boggio, J. M. ; Guimaraes, A. ; Callegari, F. A.; Marconi, J.D.; Rocha, M. L.; de Barros, M. R. X. \& Fragnito, H.L. (2004a). Parametric amplifier for mid-span phase conjugation with simultaneous compensation of fiber loss and chromatic dispersion at $10 \mathrm{~Gb} / \mathrm{s}$. Microwave and Optical Technology Letters, v. 42, p. 503-505.

Chavez Boggio, J.M.; Callegari, F.A.; Marconi, J.D.; Guimarães, A.; Fragnito, H.L. (2004b). Influence of zero-dispersion wavelength variations on cross-talk in single-pumped fiber optic parametric amplifiers. Optics Communications, vol. 242, no. 4-6, pp. 471-478.

Chavez Boggio, J.M.; Guimarães, A.; Callegari, F.A.; Marconi, J.D. \& Fragnito, H.L. (2005a). $\mathrm{Q}$ penalties due to pump phase modulation and pump RIN in fiber optic parametric amplifiers with non-uniform dispersion. Optics Communications, vol. 249, no. 4-6, pp. 451-472.

Chavez Boggio, J.M.; Marconi, J.D.; Fragnito, H.L. (2005b). Double-pumped fiber optical parametric amplifier with flat gain over 47-nm bandwidth using a conventional dispersion-shifted fiber. IEEE Photonics Technology Letters, vol. 17, no.9, pp.1842-1844.

Chen, Y. (1989). Four-wave mixing in optical fibers: exact solution. Journal of Optical Society of America B, vol. 6, no.11, pp. 1986-1993.

Fagotto, E.A.M. \& Abbade, M.L.F. (2010). All-optical demultiplexing of 4-ASK optical signals with four-wave mixing optical gates. Optics Communications, vol. 283, no. 6, pp. 1102-1109.

FAPESP. (n.d.). KyaTera, July 25 (2011), Available from: http://www.kyatera.fapesp.br/ Gloge, D. (1971). Weakly Guiding Fibers. Appl. Opt., 10, vol. 10, 2252-2258.

Grudinin, A.B.; Dianov, E.M.; Korobkin, D.V.; Prokhorov, A.M.; Serkin, V.N. \& Khaidarov, D.V. (1987). Decay of femtosecond pulses in single-mode optical fibers. Journal of Experimental and Theoretical Physics Letters, vol. 46, no. 11, pp. 221-225.

Hansryd, J. \& Andrekson, P.A. (2001). Broad-band continuous-wave-pumped fiber optical parametric amplifier with 49-dB gain and wavelength-conversion efficiency. Photonics Technology Letters, IEEE, vol.13, no.3, pp.194-196. 
Hansryd, J.; Andrekson, P.A.; Westlund, M.; Li, J. \& Hedekvist, P.O. (2002). Fiber-Based Parametric Amplifiers and Their Applications. IEEE Journal of Selected Topics in Quantum Electronics, vol. 8, (3), pp. 506-520.

Inoue, K. \& Toba, H. (1992). Wavelength conversion experiment using fiber four-wave mixing, Photonics Technology Letters, IEEE , vol.4, no.1, pp.69-72.

Jackson, J.D. (1998). Classical Electrodynamics, Wiley; 3rd Ed., New York USA.

Kalogerakis, G.; Shimizu K.; Marhic, M.E.; Wong, K.K.Y.; Uesaka, K. \& Kazovsky, L.G. (2006). High-repetition-rate pulsed-pumped fiber OPA for amplification of communication signals. Journal of Lightwave Technology., v. 24, no. 8, pp. 3021-3027.

Lu, Guo-Wei \& Miyazaki, T. (1997). Experimental Demonstration of RZ-8-APSK Generation Through Optical Amplitude and Phase Multiplexing. IEEE Photonics Technology Letters, Vol.20, no. 23, pp.1995-1997.

Marconi, J. D., Abbade M. L. F., Costa, A. L. A., Moschim, E. \& Fragnito, H.L. (2011). Experimental Analysis of All-optical 4-ASK Signal Generation through Parametric Amplification, submitted to Optics Communications.

Mishina, K., Kitagawa, S. \& Maruta, A. (2007). All-optical modulation format conversion from on-off-keying to multiple-level phase-shift-keying based on nonlinearity in optical fiber. Optics Express, vol. 15, pp. 8444-8453.

Mussot, A.; Lantz, E.; Durécu-Legrand, A.; Simonneau, C.; Bayart, D.; Maillotte, H. \& T. Sylvestre (2007). Simple method for crosstalk reduction in fiber optical parametric amplifiers. Optics Communications, vol. 275, no. 2, pp. 448-452.

Oda, S. \& Maruta, A. (2006). Two-bit all-optical analog to-digital conversion by filtering broadened and split spectrum induced by soliton effect or self-phase modulation in fiber. IEEE Journal of Selected Topics of Quantum Electronics, vol. 12, no. 2, pp. 307-314.

Olsson, B.-E.; Ohlen, P.; Rau, L.; Blumenthal, D.J. (2000). A simple and robust 40-Gb/s wavelength converter using fiber cross-phase modulation and optical filtering. IEEE Photonics Technology Letters, vol.12, no.7, pp.846-848.

Peucheret, C.; Lorenzen, M.; Seoane, J.; Noordegraaf, D.; Nielsen, C.V.; Gruner-Nielsen, L. \& Rottwitt, K. (2009). Amplitude regeneration of RZ-DPSK signals in single-pumped fiber-optic parametric amplifier. IEEE Photonics Technology Letters, vol. 21, pp. 872-874.

Ramaswamy, R.; Sivarajan, K.; Sasaki, G; (2010). Optical Networks - a practical perspective, Morgan-Kaufmann, $3^{\text {rd }}$.ed., Burlington MA, USA.

Song, S.; Allen, C.T.; Demarest, K.R. \& Hui, R. (1999). Intensity-dependent phase-matching effects on four-wave mixing in optical fibers. Jounal of Lightwave Technology, vol. 17, no. 11, pp. 2285-2290.

Stolen, R.H. \& Bjorkholm, J.E. (1982). Parametric amplification and frequency conversion in optical fibers. IEEE Journal of Quantum Electronics., vol. 18, no. 7, pp. 1062-1072.

Jamshidifar, M.; Vedadi, A. \& M. Marhic, M. E. (2010). Continuous-wave parametric amplification in bismuth-oxide fibers, Optical Fiber Technology, vol. 16, pp. 458-466.

Walklin, S. \& Conradi, J. (1999). Multilevel signaling for increasing the reach of $10 \mathrm{~Gb} / \mathrm{s}$ lightwave systems. Journal of Lightwave Technology, vol. 17, no. 11, pp. 2235-2248.

$\mathrm{Xu}$, Zhaowen ; Zhou, Guangtao \& Lu, Chao. (2005). Optical 4-ASK signal generation through four-wave mixing. Proceedings of the 18th Annual Meeting of the IEEE, Lasers and Electro-Optics Society, pp.505-506, Sydney, Australia, October, 2005.

Zhou, G. T.; Xu, K.; Wu, J.; Yan, Cishuo; Su, Yikai \& Lin, J. T. (2006). Self-Pumping Wavelength Conversion for DPSK Signals and DQPSK Generation Through FourWave Mixing in Highly Nonlinear Optical Fiber. IEEE Photonics Technology Letters, Vol.18, no.22, pp. 2389-2391. 


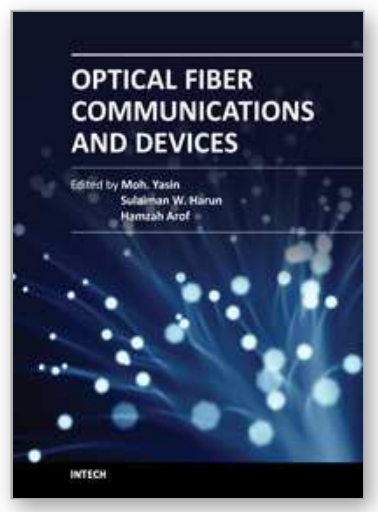

\author{
Optical Fiber Communications and Devices \\ Edited by Dr Moh. Yasin
}

ISBN 978-953-307-954-7

Hard cover, 380 pages

Publisher InTech

Published online 01, February, 2012

Published in print edition February, 2012

This book is a collection of works dealing with the important technologies and mathematical concepts behind today's optical fiber communications and devices. It features 17 selected topics such as architecture and topologies of optical networks, secure optical communication, PONs, LANs, and WANs and thus provides an overall view of current research trends and technology on these topics. The book compiles worldwide contributions from many prominent universities and research centers, bringing together leading academics and scientists in the field of photonics and optical communications. This compendium is an invaluable reference edited by three scientists with a wide knowledge of the field and the community. Researchers and practitioners working in photonics and optical communications will find this book a valuable resource.

\title{
How to reference
}

In order to correctly reference this scholarly work, feel free to copy and paste the following:

Marcelo L. F. Abbade, Jorge D. Marconi, Eric A. M. Fagotto, Felipe R. Barbosa, André L. A. Costa, Iguatemi E. Fonseca and Edson Moschim (2012). All-Optical Amplitude Multiplexing Through Fiber Parametric Interaction Between Binary Signals, Optical Fiber Communications and Devices, Dr Moh. Yasin (Ed.), ISBN: 978-953-307954-7, InTech, Available from: http://www.intechopen.com/books/optical-fiber-communications-and-devices/alloptical-amplitude-multiplexing-through-fiber-parametric-interaction-between-binary-signals

\section{INTECH}

open science | open minds

\author{
InTech Europe \\ University Campus STeP Ri \\ Slavka Krautzeka 83/A \\ 51000 Rijeka, Croatia \\ Phone: +385 (51) 770447 \\ Fax: +385 (51) 686166 \\ www.intechopen.com
}

\author{
InTech China \\ Unit 405, Office Block, Hotel Equatorial Shanghai \\ No.65, Yan An Road (West), Shanghai, 200040, China \\ 中国上海市延安西路65号上海国际贵都大饭店办公楼 405 单元 \\ Phone: +86-21-62489820 \\ Fax: +86-21-62489821
}


(C) 2012 The Author(s). Licensee IntechOpen. This is an open access article distributed under the terms of the Creative Commons Attribution 3.0 License, which permits unrestricted use, distribution, and reproduction in any medium, provided the original work is properly cited. 